\title{
ON LEVEL SETS WITH "NARROW THROATS" IN LINEAR DIFFERENTIAL GAMES
}

\author{
SERGEY S. KUMKOV*, VALERY S. PATSKO ${ }^{\dagger}$ \\ Institute of Mathematics and Mechanics, \\ Ural Branch of Russian Academy of Sciences, \\ S.Kovalevskaya str., 16, Ekaterinburg, 620219, Russia \\ JOSEF SHINAR ${ }^{\ddagger}$ \\ Faculty of Aerospace Engineering, Technion - \\ Israel Institution of Technology, Haifa, 32000, Israel
}

\begin{abstract}
Examples with zero-sum linear differential games of fixed terminal time and a convex terminal payoff function depending on two components of the phase vector are considered. Such games can have an indifferent zone with constant value function. The level set of the value function associated with the indifferent zone is called the "critical" tube. In the selected examples, the critical tube and the neighboring level sets exhibit "narrow throats". Presence of such throats requires extremely precise computations for constructing the level sets. The paper presents different forms of critical tubes with narrow throats and indicates the combinations of problem parameters that can produce them.
\end{abstract}

\section{Introduction}

The solution of a two-person zero-sum differential game is given by the triplet of the players' optimal strategies and the value function (Isaacs, 1965). The value function, which can be represented by its level sets, is the solution of the corresponding Hamilton-Jacobi-Isaacs partial differential equation and its knowledge allows deriving the respective optimal strategies. Solving this equation for a general zero-sum differential game is very complicated. Fortunately, linear zero-sum differential games yield in many cases simpler numerical and, sometimes, analytical solutions. Games with terminal payoff and fixed terminal time belong to this category. Their solution can be well illustrated by the ensemble of the level sets of the value function, which are also the loci of optimal trajectories.

The paper deals with examples of linear differential games of fixed terminal time and a convex terminal payoff function depending on two components of the phase vector with constrained controls. Such a game can be transformed (Krasovskii and Subbotin (1988), pp. 89-91) to a two-dimensional "equivalent" game, for which the solution can be visualized by the level sets of the value function and their time-sections. This class of games is characterized by the eventual existence of an

\footnotetext{
*E-mail: 2445@mail.ur.ru

†E-mail: patsko@imm.uran.ru

${ }_{\ddagger}$ E-mail: aer4301@aerodyne.technion.ac.il
} 
indifferent zone of the game space, where the value function is constant and the optimal strategies are arbitrary. The level set of the value function associated with the indifferent zone is called a "critical" tube. In the examples, the critical tube and the neighboring level sets exhibit "narrow throats". The narrow throat of the critical tube is located where the tube has, at least in one direction, zero width, as indicated by its name. The form of the critical level set and the neighboring ones near the throat can be rather complex. Presence of such throats requires extremely precise computations for constructing the level sets.

A numerical method for constructing level sets of the value function in linear differential games with fixed terminal time was developed (Isakova et al. (1984), Patsko (1996)) at the Institute of Mathematics and Mechanics (Russian Academy of Sciences, Ekaterinburg, Russia). Simultaneously, specialized visualizational programs were created (Averbukh et al. (1999)). The existing software allows illustrating the different forms of critical tubes with narrow throats and analyzing their dependence on the problem parameters.

In this paper, four examples of differential games are considered. All the examples belong to a class of problems called in Russian mathematical literature on differential games the "generalized L.S.Pontryagin's test example" (Pontryagin (1972), Mezentsev (1972), Nikol'skii (1984)). The first of these examples is based on previously published works (Shinar et al. (1984), Shinar and Zarkh (1996)) motivated by the problem of air-to-air interception. Three other examples were published in (Kumkov et al. (2000), Kumkov and Patsko (2000)).

There are several publications describing other numerical methods for constructing level sets of the value function in linear differential games with fixed terminal time (Ushakov (1981), Subbotin and Patsko (1984), Taras'ev et al. (1988), Grigorenko et al. (1993), Bardi and Dolcetta (1997), Kurzhanski and Valyi (1997), Cardaliaguet et al (1999), Polovinkin et al (2001)). The examples presented in this paper can be used for testing the accuracy and efficiency of such methods.

The structure of the paper is the following. In the next section, a formulation of zero-sum linear differential games with fixed terminal time is presented, starting with the form of the generalized L.S.Pontryagin's test example and followed by a description of the transformation of the original game to a reduced order equivalent game. In Section 3, the solution of the equivalent game is presented and the presence of a narrow throat is explained by a simple example. In Section 4, the numerical method used for constructing the level set of the value function is given. In Sections 5-6 the different examples are presented. The lessons learned from this study are discussed in Section 7, followed by some concluding remarks.

\section{Linear Differential Games with Fixed Terminal Time}

We start with the "generalized L.S.Pontryagin's test example" formulation of a zero-sum linear differential game with fixed terminal time:

$$
\begin{array}{ll}
x^{(k)}+a_{k-1} x^{(k-1)}+\ldots+a_{1} \dot{x}+a_{0} x=u, & u \in P, \\
y^{(s)}+b_{s-1} y^{(s-1)}+\ldots+b_{1} \dot{y}+b_{0} y=v, & v \in Q .
\end{array}
$$

Here, $x$ and $y$ are the position coordinates of two objects in Euclidian space, and $u$ and $v$ are their controls, constrained to respective compact sets. The coefficients 
in the equations are assumed constant. The game terminates at a given prescribed time $T$.

The game has a terminal payoff function depending on the distance between the two players at $T$ :

$$
J=\varphi(x(T), y(T))=|x(T)-y(T)| .
$$

This function is minimized by the first player (with control $u$ ) and maximized by the second (with control $v$ ).

If the vectors $x$ and $y$ are only two dimensional, the change of variables

$$
\begin{array}{ll}
z_{1}=x_{1}-y_{1}, & z_{2 k+1}=y_{1}, \\
z_{2}=x_{2}-y_{2}, & z_{2 k+2}=y_{2}, \\
z_{3}=\dot{x}_{1}, & z_{2 k+3}=\dot{y}_{1}, \\
z_{4}=\dot{x}_{2}, & z_{2 k+4}=\dot{y}_{2}, \\
z_{5}=\ddot{x}_{1}, & z_{2 k+5}=\ddot{y}_{1}, \\
z_{6}=\ddot{x}_{2}, & z_{2 k+6}=\ddot{y}_{2}, \\
\ldots \ldots \ldots \ldots \ldots & \ldots \ldots \ldots \cdots \cdots \\
z_{2 k-1}=x_{1}^{(k-1)}, & z_{2(k+s)-1}=y_{1}^{(s-1)}, \\
z_{2 k}=x_{2}^{(k-1)}, & z_{2(k+s)}=y_{2}^{(s-1)}
\end{array}
$$

transforms the system $(2.1)$ to the standard form

$$
\begin{aligned}
& \dot{z}=A z+B u+C v, \\
& z \in R^{2(k+s)}, u \in P, v \in Q
\end{aligned}
$$

with constant matrices $A, B, C$ and the payoff function

$$
\varphi\left(z_{1}(T), z_{2}(T)\right)=\sqrt{z_{1}^{2}(T)+z_{2}^{2}(T)} .
$$

For some particular cases, some more convenient variable exchange can exist.

The representation $(2.3),(2.4)$ is a particular case of the standard linear differential game

$$
\dot{z}=A(t) z+B(t) u+C(t) v, \quad t \in\left[t_{0}, T\right], z \in R^{n}, u \in P, v \in Q .
$$

with bounded controls, fixed terminal time $T$ and convex terminal payoff function $\varphi\left(z_{i}(T), z_{j}(T)\right)$ depending on two components $z_{i}, z_{j}$ of the state vector $z$.

By using the transformation (Krasovskii and Subbotin (1988), pp. 89-91)

$$
\xi(t)=K \Phi(T, t) z(t)
$$

where $\Phi(T, t)$ is the fundamental Cauchy (transition) matrix of the original homogeneous system of (2.5) and $K$ is a constant rectangular $2 \times n$ matrix extracting the $i$-th and $j$-th rows from an $n \times n$ matrix, the standard form (2.5) of the original game can be reduced to a two-dimensional "equivalent" differential game. The term "equivalent" means that if the states $\xi$ and $z$ satisfy $(2.6)$, the value $\mathcal{V}(t, \xi)$ of the value function $\mathcal{V}$ of the new game is equal to the value $V(t, z)$ of the value function $V$ of the original game. Moreover, the optimal strategies of the new game are also identical to the optimal strategies of the original game. 
The dynamics of the equivalent two-dimensional game is independent of the state vector $\xi$ :

$$
\begin{aligned}
& \dot{\xi}=D(t) u+E(t) v, \\
& t \in\left[t_{0}, T\right], \quad \xi \in R^{2}, u \in P, v \in Q \\
& D(t)=K \Phi(T, t) B(t), \quad E(t)=K \Phi(T, t) C(t)
\end{aligned}
$$

with the payoff function $J=\varphi\left(\xi_{1}(T), \xi_{2}(T)\right)$. If in the original game the payoff function is given by $(2.2)$, then the payoff function of the equivalent game takes form $J=\varphi(\xi(T))=|\xi(T)|$.

Let us call the sets $\mathcal{P}(t)=D(t) P$ and $\mathcal{Q}(t)=E(t) Q$ the vectograms of the first and second players at the instant $t$. The vectogram $\mathcal{P}(t)(\mathcal{Q}(t))$ describes the collection of all possible contributions of the first (second) player to the speed of the system. Respective location of the sets $\mathcal{P}(t)$ and $\mathcal{Q}(t)$ characterizes advantage of some or other player at different directions. To explain qualitatively the shape of the time sections $\mathcal{W}_{c}(t)$ of a level set $\mathcal{W}_{c}$ of the value function $(t, \xi) \mapsto \mathcal{V}(t, \xi)$ in the equivalent game, it is useful to see the tubes of the set $-\mathcal{P}(t)$ and $-\mathcal{Q}(t)$ enrolled in the backward time. Let us call these tubes the tubes of vectograms of the first and second player and denote them by $\mathcal{P}$ and $\mathcal{Q}$ respectively.

In the case when the original game dynamics is of the (2.1) type, the matrices $D(t)$ and $E(t)$ have the form $D(t)=\zeta(t) \cdot I_{2}, E(t)=\eta(t) \cdot I_{2}$, where $I_{2}$ is a unit matrix $2 \times 2$, and $\zeta(t)$ and $\eta(t)$ are scalar functions of time. So, for this class of games, players' vectograms are computed as $\mathcal{P}(t)=\zeta(t) P$ and $\mathcal{Q}(t)=\eta(t) P$.

\section{Solving the Equivalent Game}

The first step in the solution of the equivalent game according to the Isaacs method is by using the necessary conditions of game optimality. The Hamiltonian function of the game is

$$
H(\xi, \lambda, t, u, v)=\lambda^{\prime}[D(t) u+E(t) v],
$$

where $\lambda(t)$ represents (along optimal candidate trajectories) the gradient of the cost function and has to satisfy the adjoint equation

$$
\dot{\lambda}=-\frac{\partial H(\xi, \lambda, t, u, v)}{\partial \xi}=0
$$

and the corresponding transversality condition

$$
\lambda(T)=\operatorname{grad} J=\frac{\xi(T)}{|\xi(T)|} .
$$

Relations (3.8) and (3.9) yield

$$
\lambda(t)=\lambda(T)=\frac{\xi(T)}{|\xi(T)|}=\alpha^{*}
$$

where $\alpha^{*}$ is a constant unit vector on each optimal trajectory. 
The candidate optimal strategy pair $u^{*}$ and $v^{*}$ can now be determined by

$$
\begin{aligned}
& u^{*}(t)=\underset{u \in P}{\arg \min } \max H(\xi, \lambda(t), t, u, v)=\underset{v \in Q}{\arg \min } \lambda^{\prime}(t) D(t) u=\underset{u \in P}{\arg \min } \alpha^{* \prime} D(t) u, \\
& v^{*}(t)=\underset{v \in Q}{\arg \max } \min _{u \in P} H(\xi, \lambda(t), t, u, v)=\underset{v \in Q}{\arg \max } \lambda^{\prime}(t) E(t) v=\underset{v \in Q}{\arg \max } \alpha^{* \prime} E(t) v,
\end{aligned}
$$

where the explicit expressions depend on the control constraints of the game. Since $\alpha^{*}$ is a constant vector, the equations of the candidate optimal trajectories can be directly integrated backwards in time from any point at the terminal time $T$.

Each level sets of the value function are generated by the family of such candidate optimal trajectories, all starting (in reverse time) at different points on a circle at the terminal time $T$. If all candidate optimal trajectories, generated by backwards integration in time, succeed to fill the entire equivalent game space without intersecting each other, the sufficiency conditions of game optimality (Isaacs (1965)) are satisfied by (3.11). In this case, we can obtain level sets of the value function and the value function itself.

Unfortunately, in most cases such completely "regular" solutions do not exist for two reasons. Singularities in differential games are rather a rule, not an exception. The candidate optimal trajectories frequently intersect and create singular surfaces of the game space, where at least one component of $\lambda(t)$ is discontinuous. This means that the solution (3.10) is not valid any more. If the game space cannot be filled by regular candidate optimal trajectories, the empty regions also involve some singularities. In order to continue the solution, the type of the singularities has to be analyzed, which is a very complex task (Melikyan and Shinar (2000)). In the linear differential games specified in Section 2, the optimal game solution is based on the decomposition of the equivalent game space to regions of different optimal controls. One of the regions is called the regular zone and the controls derived by (3.11) are optimal there. Some of the empty regions become "indifferent" zones, where the value is constant and the optimal strategies are arbitrary.

This situation can be illustrated, if the control constraints are circular (with radii $u_{\max }$ and $v_{\max }$ respectively), by a very simple example having an analytical solution. In this case, the game solution will be identical in every plane of arbitrary direction (for example, in the plane $\xi_{2}=0$ ). Therefore, in the sequel a planar case is considered, as shown in Figure 1.

The candidate optimal strategies (3.11) in this planar case become

$$
u_{1}^{*}=-u_{\max } \operatorname{sign}\{\zeta(t)\} \operatorname{sign}\left\{\xi_{1}(T)\right\}, \quad v_{1}^{*}=v_{\max } \operatorname{sign}\{\eta(t)\} \operatorname{sign}\left\{\xi_{1}(T)\right\} .
$$

The trajectory dynamics in this plane can be written as

$$
\begin{gathered}
\dot{\xi}_{1}^{*}=\zeta(t) u_{1}^{*}+\eta(t) v_{1}^{*}=-u_{\max }|\zeta(t)| \operatorname{sign}\left\{\xi_{1}(T)\right\}+v_{\max }|\eta(t)| \operatorname{sign}\left\{\xi_{1}(T)\right\}= \\
\left(-u_{\max }|\zeta(t)|+v_{\max }|\eta(t)|\right) \operatorname{sign}\left\{\xi_{1}(T)\right\}=\Gamma(t) \operatorname{sign}\left\{\xi_{1}(T)\right\} .
\end{gathered}
$$

Thus, all candidate optimal trajectories differ from each other by a shift along the axis $\xi_{1}$ depending on the initial point $\xi_{1}(T)$. Assume that the function $\Gamma(t)$ is positive for $t<T$ (at the neighborhood of $T$ ) and changes its sign only once in 


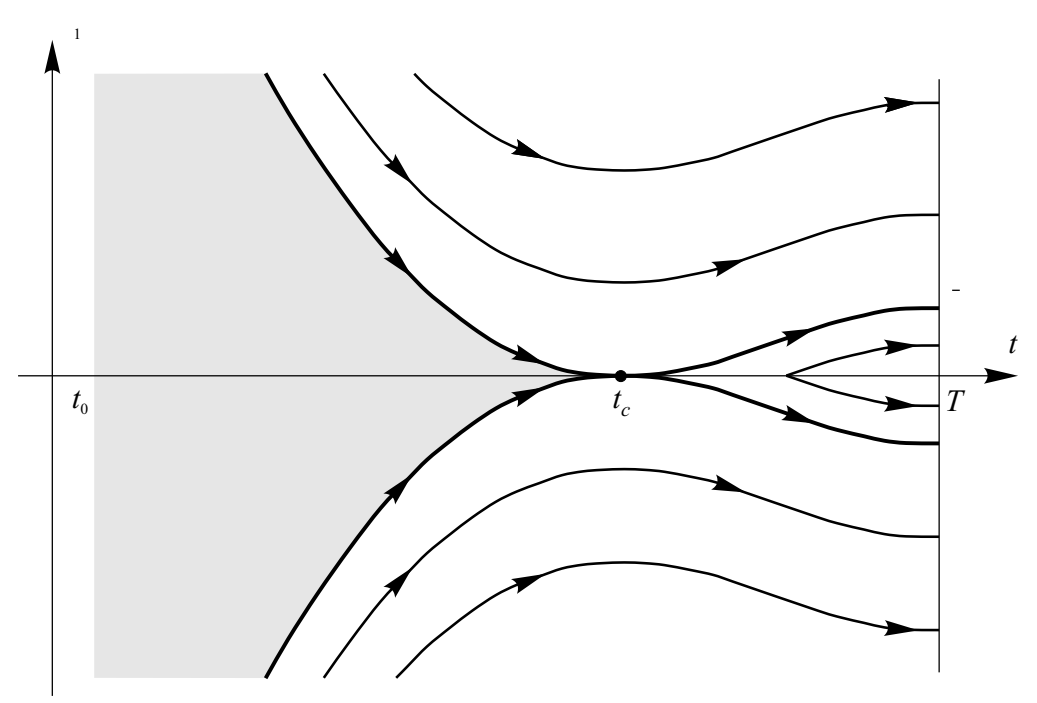

Figure 1: Presence of a throat and an indifferent zone in the problem with circular vectograms. (The indifferent zone is drawn in gray.)

the time interval $t_{0}<t<T$. Then a candidate optimal trajectory terminating at $\xi_{1}(T)>0$ can reach (backwards) the time axis and ceases to be optimal. Thus, its backwards integration cannot continue. By increasing the value of $\xi_{1}(T)$ up to a value $\bar{\xi}$, one will arrive to a candidate optimal trajectory that reaches the $t$ axis tangentially at an instant $t=t_{c}$ such that $\Gamma\left(t_{c}\right)=0$. This trajectory can be integrated further backwards in time (see Figure 1).

Since the solution is identical in all the planes that include the $t$ axis, the family of such trajectories starting on the circle $|\xi|=\bar{\xi}$ generates the "critical" level set of the game value function and the region near to the point of tangency is called the "throat". The instant associated with the point of tangency can be named as "instant of degeneration of the level set". This phenomenon leads to the decomposition of the equivalent game space.

All trajectories starting in the region between the critical trajectories associated with $t<t_{c}$, can leave it (in forward time) only at the throat. From this point, further motion with $t>t_{c}$ must continue along one of the critical trajectories yielding the same cost. Therefore, the optimal strategies in the entire region are arbitrary and value function of the game is constant. This is a particular singular region of the game, called the "indifferent" zone (Melikyan and Shinar (2000)). The point associated with "instant of degeneration of the level set" is a singular dispersal point and the section $t>t_{c}$ of the $t$ axis is a dispersal line of the game, both dominated by the second (maximizing) player.

In the general case, $\Gamma(t)$ can change it sign more than once and this can lead to the existence of several throats of one level set or several indifferent zones generated by different level sets.

In the case with circular control constraints, all time sections of the level sets of 
the value function are circular and the narrow throat is also circular around a point in the time axis. However, if the circular symmetry is disturbed, for example by elliptical control constraints, the throat becomes elongated in one direction. In such cases, the time sections of the critical tube can be of a very complicated structure and the construction of the level sets of the value function becomes a very complex task that requires extremely precise numerical methods. The complexity of the form of the critical level set and its neighbors is illustrated in several examples in the sequel.

\section{Backward Procedure for Constructing Level Sets}

Now, let us describe the algorithm (Isakova et al. (1984)) for constructing level sets of the value function (maximal stable bridges) of games of the type (2.5), (2.7). All constructions are made in the equivalent coordinates with the transformation to the equivalent game being done numerically and in automatic way. The procedures created are of the backward type and can be treated as the dynamic programming principle applied to differential games.

To do the numerical construction, let us take a sequence of instants $t_{0}<t_{1}<$ $t_{2}<\ldots<t_{N-1}<t_{N}=T$ in the time interval $\left[t_{0}, T\right]$ of the game. Uniformity of the grid is unessential. For a given constant $c$, the result of the procedure is a collection of sets $\boldsymbol{W}_{c}\left(t_{i}\right)$ corresponding to the chosen time instants $t_{i}$ and approximating the sections $\mathcal{W}_{c}\left(t_{i}\right)$ of the level set $\mathcal{W}_{c}=\{(t, \xi): \mathcal{V}(t, \xi) \leq c\}$ of the value function $\mathcal{V}$ of the game (2.7) at these instants.

Change the dynamics of the game (2.7) by a piecewise-constant dynamics

$$
\dot{\xi}=\boldsymbol{D}(t) u+\boldsymbol{E}(t) v, \quad \boldsymbol{D}(t)=D\left(t_{i}\right), \quad \boldsymbol{E}(t)=E\left(t_{i}\right), \quad t \in\left[t_{i}, t_{i+1}\right) .
$$

Instead of the original constraints $P$ and $Q$ for the controls of the players, let us consider their polyhedral approximations $\boldsymbol{P}$ and $\boldsymbol{Q}$. Let $\hat{\varphi}$ be the approximating payoff function. It is defined so that for any number $c$, its level set $\boldsymbol{M}_{c}=\{\xi$ : $\hat{\varphi}(\xi) \leq c\}$ is a convex polygon close in Hausdorff metrics to the level set $\mathcal{M}_{c}$ of the original payoff function.

The approximating game (4.12) is chosen such that in each step $\left[t_{i}, t_{i+1}\right]$ of the backward procedure we deal with a game with simple motions (Isaacs (1965)) and polyhedral convex constraints for the players' controls. At the first step $\left[t_{N-1}, t_{N}\right]=$ $\left[t_{N-1}, T\right]$, a solvability set $\boldsymbol{W}_{c}\left(t_{N-1}\right)$ for a game of homing with target set $\boldsymbol{W}_{c}\left(t_{N}\right)=$ $\boldsymbol{M}_{c}$ is constructed. Here, the first player tries to guide the system to the set $\boldsymbol{W}_{c}\left(t_{N}\right)$ at the instant $t_{N}$, and the second one opposes this. Continuing in the same way, a set $\boldsymbol{W}_{c}\left(t_{N-2}\right)$ is constructed on the base of $\boldsymbol{W}_{c}\left(t_{N-1}\right)$, and so on. As a result, we obtain a collection of convex polygons $\boldsymbol{W}_{\boldsymbol{c}}\left(t_{i}\right)$ approximating (Ponomarev and Rozov (1978), Botkin (1982), Polovinkin et al. (2001)) the sections $\mathcal{W}_{c}\left(t_{i}\right)$ of the level set $W_{c}$ of the value function of the game (2.7) in Hausdorff metrics. An algorithm for a posteriori estimating the numerical construction error is developed (Botkin and Zarkh (1984)).

The procedure of pass from the section $\boldsymbol{W}_{c}\left(t_{i+1}\right)$ to the next one $\boldsymbol{W}_{c}\left(t_{i}\right)$ is described in terms of support functions of the sets under consideration. Recall that the value $\rho(l, A)$ of the support function of a convex closed set $A$ on the vector $l$ is 
calculated by formula

$$
\rho(l, A)=\max _{a \in A} l^{\prime} a,
$$

where the prime denotes transposition.

The support function $l \mapsto \rho\left(l, \boldsymbol{W}_{c}\left(t_{i}\right)\right)$ of the polygon $\boldsymbol{W}_{c}\left(t_{i}\right)$ coincides (Pschenichnyi and Sagaidak (1970)) with convex hull of the function

$$
\begin{array}{r}
\gamma\left(l, t_{i}\right)=\max _{\xi \in \boldsymbol{W}_{c}\left(t_{i+1}\right)} l^{\prime} y+\Delta \max _{u \in \boldsymbol{P}} l^{\prime}\left(-D\left(t_{i}\right) u\right)+\Delta \min _{v \in \boldsymbol{Q}} l^{\prime}\left(-E\left(t_{i}\right) v\right)= \\
=\rho\left(l, \boldsymbol{W}_{c}\left(t_{i+1}\right)\right)+\Delta \rho\left(l,-D\left(t_{i}\right) \boldsymbol{P}\right)-\Delta \rho\left(l, E\left(t_{i}\right) \boldsymbol{Q}\right) .
\end{array}
$$

The function $\gamma\left(\cdot, t_{i}\right)$ is positively homogeneous and piecewise-linear (because the support functions of the polygons $\boldsymbol{W}_{c}\left(t_{i+1}\right),-D\left(t_{i}\right) \boldsymbol{P}$ and $E\left(t_{i}\right) \boldsymbol{Q}$ are of this type). The property of local convexity can be violated only on boundary of linearity cones of the function $\rho\left(\cdot, E\left(t_{i}\right) \boldsymbol{Q}\right)$, that is, on boundary of cones generated by normals to neighbor edges of the polygon $E\left(t_{i}\right) \boldsymbol{Q}$. This fact is useful for developing fast and effective convexification algorithm (Isakova et al. (1984), Patsko (1996)).

\section{A Pursuit-Evasion Game with Elliptical Constraints}

\subsection{Air-to-air Interception and the Corresponding Differential Game}

In the works Shinar et al. (1984), Shinar and Zarkh (1996), and Melikyan and Shinar (2000), three-dimensional air-to air interception problems were formulated as pursuit-evasion games. The pursuer is the interceptor missile, while the evader is a maneuverable aerial target (an aircraft or another missile). The natural cost function of the game is the distance of closest approach, the miss distance, to be minimized by the pursuer and maximized by the evader. For sake of simplicity, point mass models with constant velocities $\left(V_{P}\right)_{\mathrm{col}},\left(V_{E}\right)_{0}$ were selected. (Such a difference of denotations is due to the velocity of the evader is taken by the

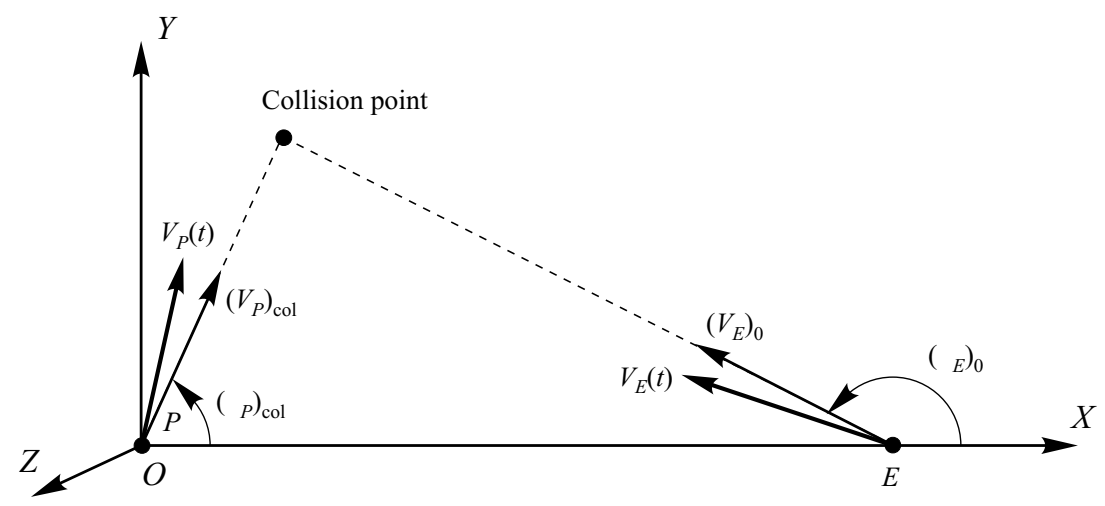

Figure 2: The system of coordinates in the problem of three-dimensional pursuit. The actual vectors of $V_{P}(t)$ and $V_{E}(t)$ slightly differ during the engagement from the nominal values $\left(V_{P}\right)_{\text {col }}$ and $\left(V_{E}\right)_{0}$, respectively 
pursuer from some outer sources, and the velocity of the pursuer is set by himself to provide the nominal collision.) The lateral accelerations of both players, normal to the respective velocity vectors, have constant bounds $a_{P}$ and $a_{E}$. The evader controls its maneuver with ideal dynamics, while the pursuer's maneuver dynamics is represented by a first order transfer function with the time constant $\tau_{P}$.

The origin of the Cartesian coordinate system is collocated with the pursuer. The direction of the $X$-axis is along the initial line of sight. The $X Y$ plane is in the nominal "collision plane" determined by the initial velocity vectors of the players. The $Z$-axis completes a right handed coordinate system, as illustrated in Figure 2.

It is assumed that the initial conditions are near to a "collision course", defined by

$$
\left(V_{P}\right)_{\mathrm{col}} \sin \left(\chi_{P}\right)_{\mathrm{col}}=\left(V_{E}\right)_{0} \sin \left(\chi_{E}\right)_{0}
$$

and the actual velocity vector of the pursuer remains close to the collision requirements, satisfying

$$
\sin \left(\chi_{P}(t)-\left(\chi_{P}\right)_{\mathrm{col}}\right) \approx \chi_{P}(t)-\left(\chi_{P}\right)_{\mathrm{col}}, \quad \cos \left(\chi_{P}(t)-\left(\chi_{P}\right)_{\mathrm{col}}\right) \approx 1 .
$$

It is also assumed that the actual velocity vector of the evader will remain close enough to the initial condition satisfying

$$
\sin \left(\chi_{E}(t)-\left(\chi_{E}\right)_{0}\right) \approx \chi_{E}(t)-\left(\chi_{E}\right)_{0}, \quad \cos \left(\chi_{E}(t)-\left(\chi_{E}\right)_{0}\right) \approx 1 .
$$

Based on the small angle assumptions (5.15) and (5.16), the relative trajectories can be linearized with respect to the initial line of sight. Moreover, the relative motion in the $X$ direction can be considered as uniform. Thus, this coordinate can be replaced by the time, transforming the original autonomous three-dimensional motion to a time-varying two-dimensional motion in the $Y Z$ plane. For a given initial range, the uniform closing velocity determines the final time $T$ of the engagement. Therefore, the problem of minimizing (maximizing) the three-dimensional miss distance at a free terminal time can be changed by the minimization (maximization) of the distance in the $Y Z$ plane at the fixed terminal time of the nominal collision.

Since in general the velocity vectors of the players are not aligned with the initial line of sight, the projections of the originally circular control constraints, normal to the respective velocity vectors, become elliptical.

The equations of motion of the linearized pursuit-evasion game are

$$
\begin{array}{ll}
\ddot{x}=F, & t \in[0, T], x, y \in R^{2}, \quad u \in P, \quad v \in Q, \\
\dot{F}=-(F-u) / \tau_{P}, & \varphi(x(T), y(T))=|x(T)-y(T)|, \\
\ddot{y}=v, &
\end{array}
$$

where $x$ and $y$ are the positions of the players in the plane normal to the initial line of sight. Eliminating $F$ in the dynamics (5.17) and denoting $\alpha=1 / \tau_{P}$ yields

$$
\dddot{x}+\alpha \ddot{x}=\alpha u, \quad \ddot{y}=v,
$$

which is in the form of a typical "generalized L.S.Pontryagin's test example". 
To reduce the dynamics (5.18) to the form (2.5), a variable change

$$
\begin{array}{lll}
z_{1}=x_{1}-y_{1}, & z_{2}=\dot{x}_{1}-\dot{y}_{1}, & z_{3}=\ddot{x}_{1}, \\
z_{4}=x_{2}-y_{2}, & z_{5}=\dot{x}_{2}-\dot{y}_{2}, & z_{6}=\ddot{x}_{2} .
\end{array}
$$

can be applied, leading to the following standard form of the game

$$
\begin{aligned}
& \dot{z}=A z+B u+C v, \\
& A=\left[\begin{array}{c|c}
A_{1} & 0 \\
\hline 0 & A_{1}
\end{array}\right], \quad A_{1}=\left[\begin{array}{ccc}
0 & 1 & 0 \\
0 & 0 & 1 \\
0 & 0 & -1 / \tau_{P}
\end{array}\right], \\
& B^{\prime}=\left(1 / \tau_{P}\right)\left[\begin{array}{lll|lll}
0 & 0 & 1 & 0 & 0 & 0 \\
\hline 0 & 0 & 0 & 0 & 0 & 1
\end{array}\right], \\
& C^{\prime}=\left[\begin{array}{ccc|ccc}
0 & -1 & 0 & 0 & 0 & 0 \\
\hline 0 & 0 & 0 & 0 & -1 & 0
\end{array}\right], \\
& u \in P=\left\{u: u^{\prime}\left[\begin{array}{cc}
1 / \cos ^{2}\left(\chi_{P}\right)_{\operatorname{col}} & 0 \\
0 & 1
\end{array}\right] u \leq a_{P}^{2}\right\}, \\
& v \in Q=\left\{v: v^{\prime}\left[\begin{array}{cc}
1 / \cos ^{2}\left(\chi_{E}\right)_{0} & 0 \\
0 & 1
\end{array}\right] v \leq a_{E}^{2}\right\}, \\
& \varphi\left(z_{1}(T), z_{4}(T)\right)=\sqrt{z_{1}^{2}(T)+z_{4}^{2}(T)} .
\end{aligned}
$$

Transformation to the equivalent game form yields

$$
\dot{\xi}=D(t) u+E(t) v, \quad t \in[0, T], \quad \xi \in R^{2}, \quad u \in P, \quad v \in Q, \quad \varphi(\xi(T))=|\xi(T)|,
$$

where

$$
\begin{aligned}
& D(t)=\zeta(t) \cdot I_{2}, \quad \zeta(t)=(T-t)+\tau_{P} e^{-(T-t) / \tau_{P}}-\tau_{P}, \\
& E(t)=\eta(t) \cdot I_{2}, \quad \eta(t)=-(T-t) .
\end{aligned}
$$

Here, $I_{2}$ is a unity matrix $2 \times 2$.

In all realistic pursuit-evasion examples, in order to make a small miss distance feasible the pursuer must have some advantage in maximum lateral acceleration in every direction. This means that the control constraint set $P$ of the pursuer completely covers the control constraint set $Q$ of the evader. In other words, the inequalities $a_{P} / a_{E}>1$ and $a_{P}\left|\cos \left(\chi_{P}\right)_{\text {col }}\right|>a_{E}\left|\cos \left(\chi_{E}\right)_{0}\right|$ describing the relations of the semiaxes of the ellipses $P$ and $Q$ have to be valid. Such an advantage allows reducing an initial launching error and overcoming long duration constant evader maneuvers. However, due to the first order dynamics of the pursuer's control function and the ideal dynamics of the evader, zero miss distance cannot be achieved against an optimally maneuvering evader. The beginning of the engagement is dominated by the maneuverability advantage of the pursuer, but the end is dominated by the ideal dynamics of the evader. Such a transition of the advantage creates the throat. 
In Shinar et al. (1984), as well as in Melikyan and Shinar (2000), the parameters of the problem were of an interception of a manned aircraft, assuming that $\left(V_{P}\right)_{\mathrm{col}}>$ $\left(V_{E}\right)_{0}$. Thus using (5.14) one obtained

$$
\left|\cos \left(\chi_{P}\right)_{\operatorname{col}}\right|>\left|\cos \left(\chi_{E}\right)_{0}\right|
$$

In Shinar and Zarkh (1996), an interception of a tactical ballistic missile was considered with $\left(V_{P}\right)_{\text {col }}<\left(V_{E}\right)_{0}$, leading to

$$
\left|\cos \left(\chi_{P}\right)_{\operatorname{col}}\right|<\left|\cos \left(\chi_{E}\right)_{0}\right| \text {. }
$$

This difference changes considerably the form of the critical tube and the associated singular surfaces.

If $\left(V_{P}\right)_{\text {col }}>\left(V_{E}\right)_{0}$, the advantage of the pursuer is maximal in the direction of $\xi_{1}$. For this reason the evader prefers to maneuver in the direction of $\xi_{2}$, unless the initial condition in the direction of $\xi_{1}$ is quite large. (If the control constraints were circular, in the case of an initial condition in the direction of $\xi_{1}$ the evader would maneuver to this direction, but due to the elliptical constraint the optimal direction is a compromise.) In other words, the critical level set contracts more in the direction of $\xi_{2}$, before reaching the instant of degeneration, creating a narrow throat of the critical tube (and of its neighbors) elongated in the direction of $\xi_{1}$.

However, if $\left(V_{P}\right)_{\text {col }}<\left(V_{E}\right)_{0}$, as in Shinar and Zarkh (1996), the situation becomes much more complex. Below, the results of a numerical investigation of this case are shown.

\subsection{Results of Numerical Constructions}

Based on the data of the original problem

$$
\frac{\left(V_{E}\right)_{0}}{\left(V_{P}\right)_{\mathrm{col}}}=1.054, \quad \frac{a_{P}}{a_{E}}=1.3, \quad\left|\cos \left(\chi_{P}\right)_{\mathrm{col}}\right|=0.67, \quad\left|\cos \left(\chi_{E}\right)_{0}\right|=0.71, \quad \tau_{P}=1,
$$

in the construction, the following data were used: $\alpha=1 / \tau_{P}=1$,

$$
P=\left\{u \in R^{2}: \frac{u_{1}^{2}}{0.67^{2}}+\frac{u_{2}^{2}}{1.00^{2}} \leq 1.30^{2}\right\}, \quad Q=\left\{v \in R^{2}: \frac{v_{1}^{2}}{0.71^{2}}+\frac{v_{2}^{2}}{1.00^{2}} \leq 1\right\}
$$

Figure 3 shows a general view of the level set $\boldsymbol{W}_{\bar{c}}$ computed for the parameter $\bar{c}=$ 1.546, which is slightly greater than the critical one. Here and further, $\tau$ denotes the backward time: $\tau=T-t$. The most interesting feature of the tube is that it has a narrow throat. Moreover, the orientation of the elongation of the $t$-sections changes near the throat. An enlarged view of the throat is shown in Figure 4.

To explain this shape of the narrow throat one can consider the vectograms of the players. The vectogram tubes for this problem are shown in Figure 5. The tube $\mathcal{P}$ of vectograms of the first player is drawn in red, the tube $\mathcal{Q}$ of the second player is in green. Since, $\mathcal{Q}(t)=E(t) Q=\eta(t) I_{2} Q=\eta(t) Q$, where $\eta(\cdot)$ is described by formula $(5.20 \mathrm{~b})$, the green tube grows linearly in backward time. For the tube $\mathcal{P}$, we have $\mathcal{P}(t)=\zeta(t) P$, where $\zeta(\cdot)$ is taken from formula (5.20a). So, the red tube initially grows slower than linearly, and further starts to grow faster than linearly. 
The above mentioned difference of changing the vectograms in time is the reason that the ellipse $\mathcal{P}(\tau)$ of the vectogram of the first player becomes greater than the ellipse $\mathcal{Q}(\tau)$ of the second player. But because these ellipses have different eccentricities, the first player ellipse starts to cover ellipse of the second player

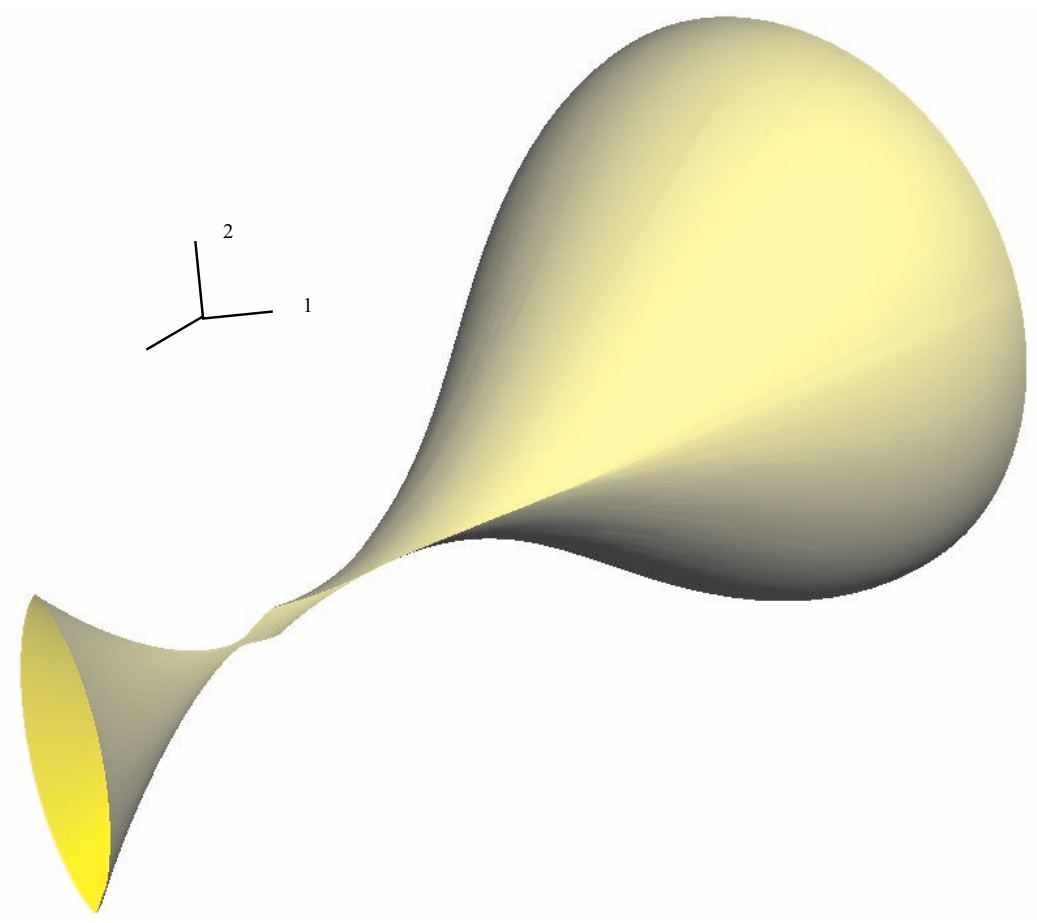

Figure 3: General view of the level set of the value function with a narrow throat

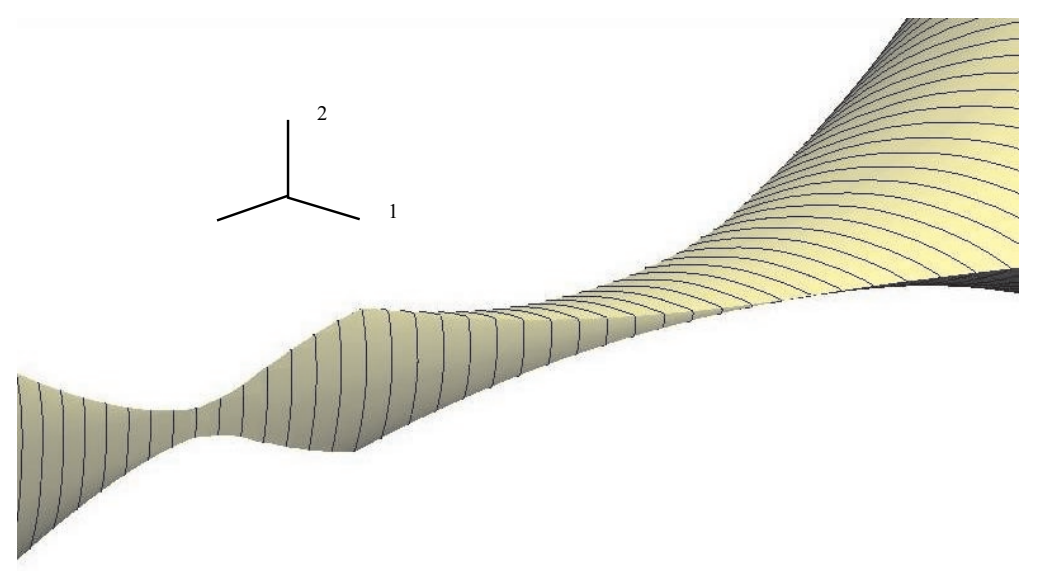

Figure 4: Enlarged view of the narrow throat 


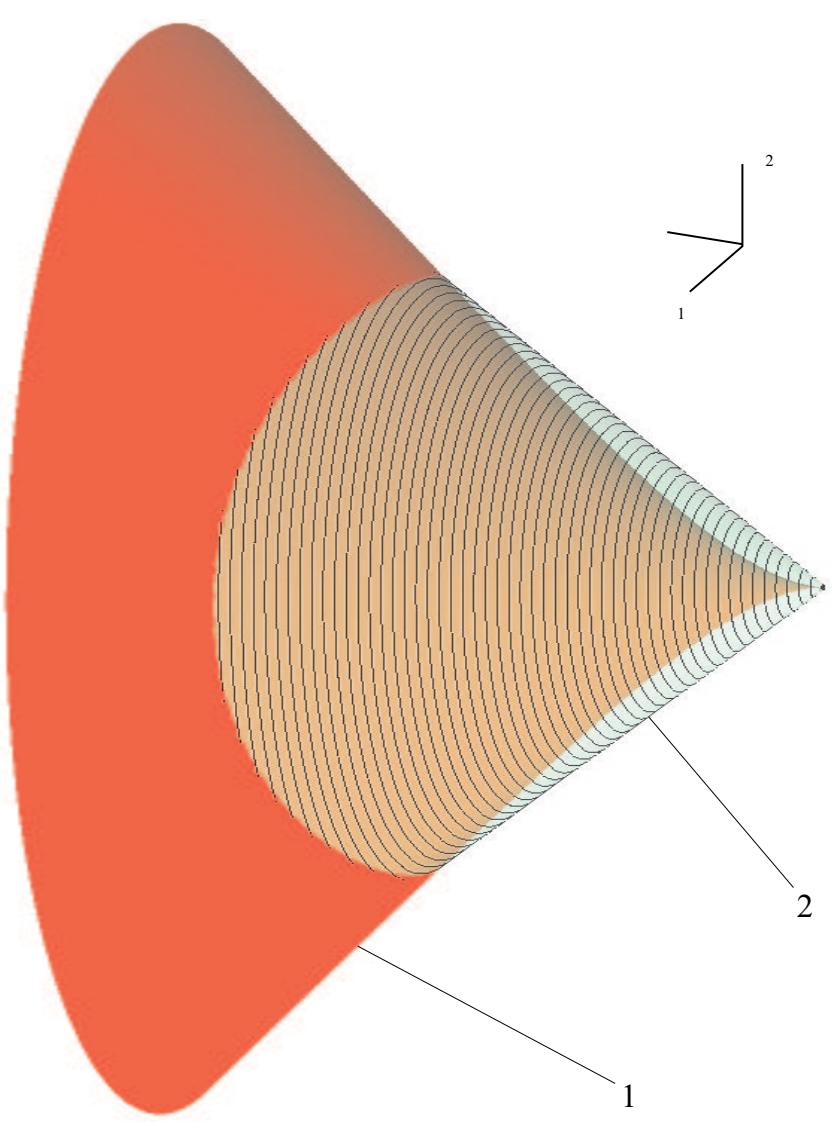

Figure 5: General view of the vectogram tubes of the first (1) and second (2) players. The vectogram tube of the second player is transparent, showning contours of some sections
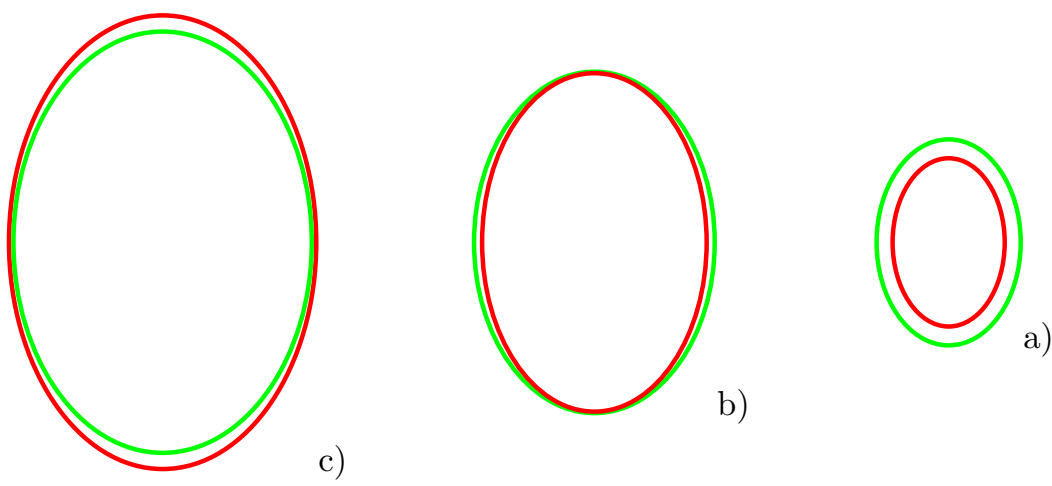

c)

Figure 6: Sections of the vectogram tubes at some time instants: a) before $\tau_{*}$, b) between $\tau_{*}$ and $\tau^{*}, \mathrm{c}$ ) after $\tau^{*}$. The vectograms of the first player are shown by the red lines, the green lines denote the vectograms of the second player 


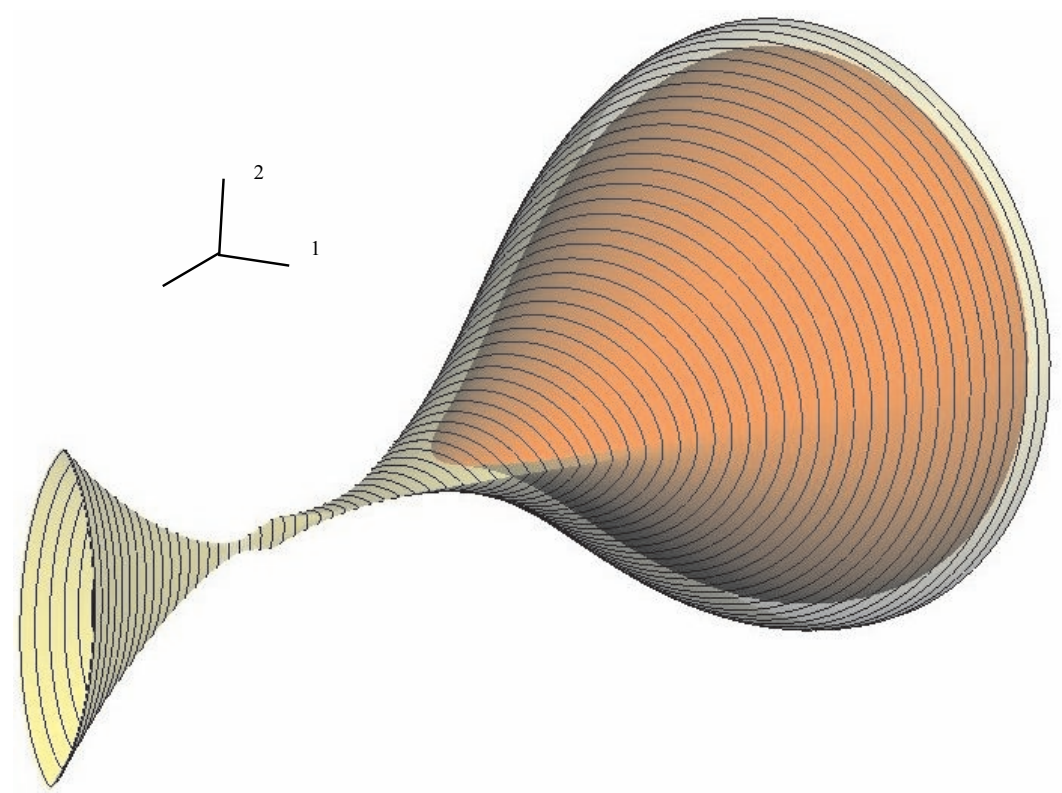

Figure 7: The level set with narrow throat for the parameter $\bar{c}=1.546$ (yellow transparent) and the level set for a less value of the parameter $c=1.48$ (red)

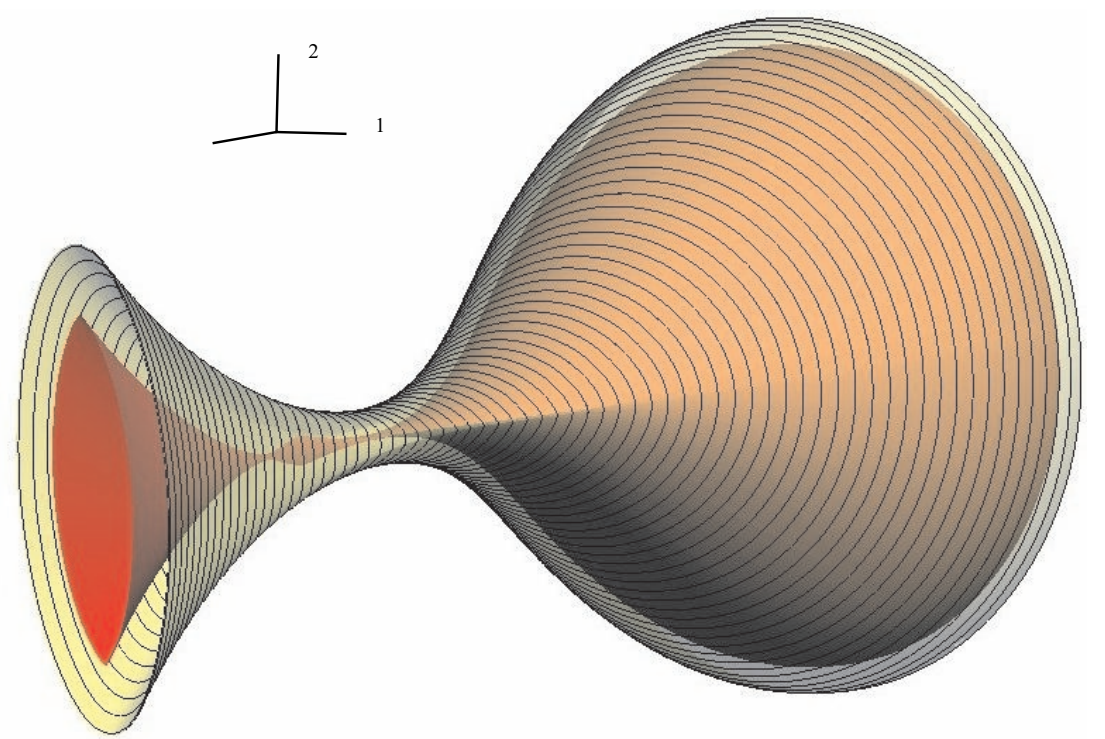

Figure 8: The level set with narrow throat for the parameter $\bar{c}=1.546$ (red) and the level set for a greater value of the parameter $c=1.67$ (yellow transparent) 
in different directions at different instants. So, before the instant $\tau_{*}=4.18$, the ellipse $\mathcal{Q}(\tau)$ includes the ellipse $\mathcal{P}(\tau)$ completely (Figure 8a). Beginning from this instant, the first player ellipse starts to cover the ellipse of the second player in the vertical direction (Figure 8b). And, finally, beginning from the instant $\tau^{*}=5.3$, the set $\mathcal{P}(\tau)$ covers the set $\mathcal{Q}(\tau)$ completely (Figure 8c).

Thus, before the instant $\tau_{*}$, the second player has complete advantage over the first one. Because in backward time the second player tries to contract the section of a level set as much as possible, the $t$-sections of the tube $\boldsymbol{W}_{c}$ diminish in the initial period of backward time (it can be seen in Figure 3). The tube contracts more in the vertical direction than in horizontal one, since the second player's advantage is greater in the vertical direction. So, at the instant $\tau_{*}$, the $t$-sections of the level set are elongated horizontally.

Finally, at the instant $\tau^{*}$, when the first player gets complete advantage, contraction of $t$-sections changes to extension. This is the instant of the most narrow section of the throat in Figure 4. Because further in backward time the first player keeps the advantage, the tube extends monotonically.

This example has been computed in the interval $\tau \in[0,7]$ of backward time. The time step $\Delta$ was taken equal to 0.01 . The circles of the level sets of the payoff function and the ellipses $P$ and $Q$ of constraints for the players' controls were approximated by 100-gons (polygons with 100 vertices).

The next two figures show the critical level set $\boldsymbol{W}_{\bar{c}}$ in comparison with level sets close to it. Figure 7 shows the tube $\boldsymbol{W}_{\bar{c}}$ (drawn in transparent yellow) and the tube computed for the value $c=1.48$ of the payoff function, which is less than the critical one. This tube is finite in time and drawn in red. In Figure 8, the critical level set $\boldsymbol{W}_{\bar{c}}$ (red) and a greater one computed for $c=1.67$ (yellow transparent). One can see that the latter has smooth boundary. These figures demonstrate that the majority of peculiarities of the value function are found near the narrow throat. This affirms the necessity of extremely accurate computations near the throat.

\section{More Examples with Narrow Throats}

Having accumulated experience in the numerical investigation of level sets with narrow throats from the work Shinar and Zarkh (1996), the first authors decided to look for another examples of this type from the class of games with the L.S.Pontryagin's generalized test example dynamics.

Interesting results of level sets construction in the generalized L.S.Pontryagin's example with the payoff function $\varphi(x(T), y(T))=|x(T)-y(T)|$ can be obtained if at least one of the sets $P, Q$ is not a circular. Suppose that the sets $P$ and $Q$ are ellipses with centers coinciding with the origin. In this case at any instant, the sets $\mathcal{P}(t)$ and $\mathcal{Q}(t)$ are ellipses, which are homothetic to the sets $P$ and $Q$, correspondingly.

In order to obtain a level set having a narrow throat, it is necessary that the advantage shifts from one player to the other. For example, in the starting interval (of backward time), the second player has the advantage, but later the first player becomes advantaged and so on. The easiest way to get change of advantage is to set the dynamics of one or both players to be oscillatory. 

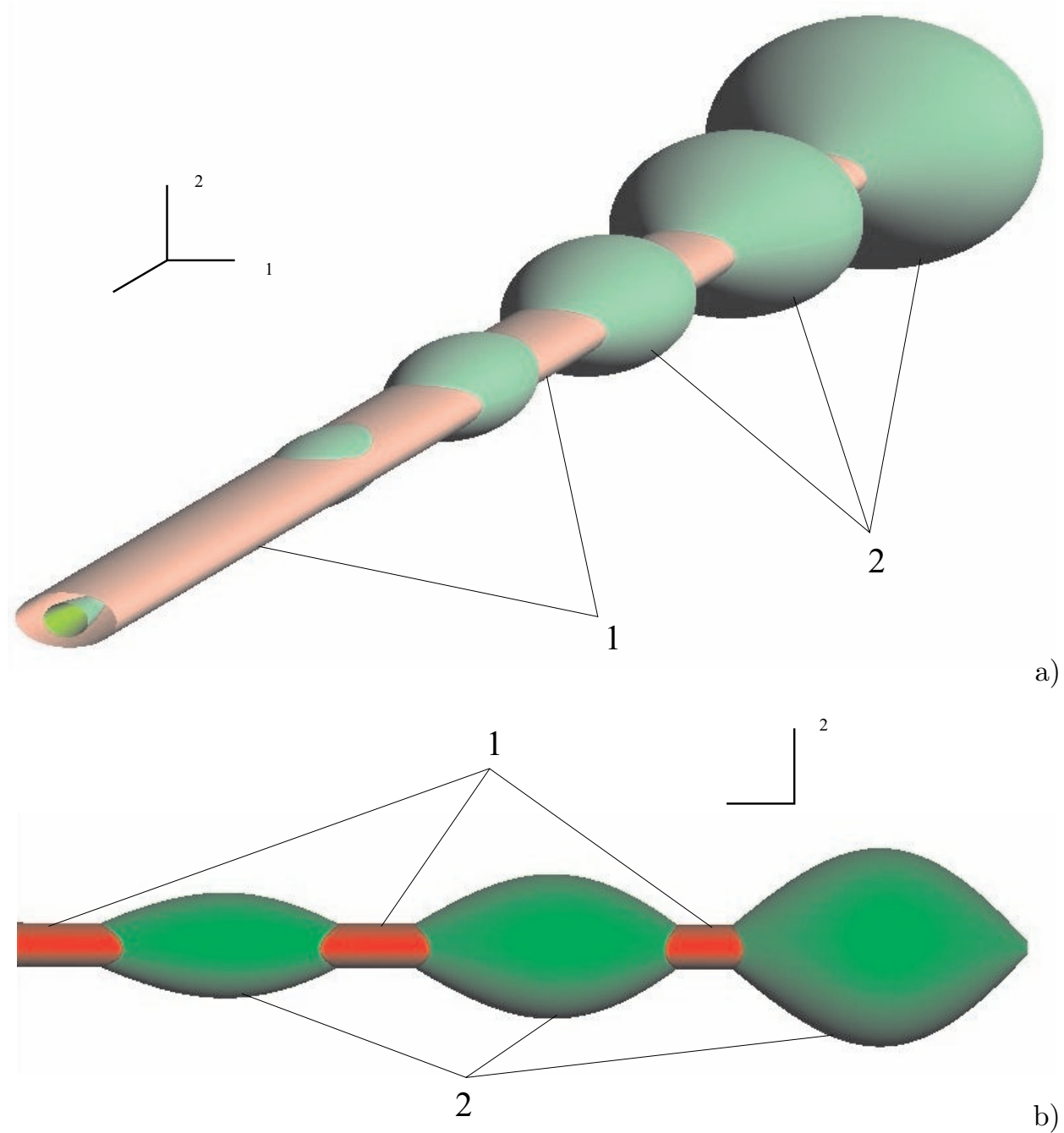

a)

b)

Figure 9: Example 1. Two views of the vectograms appearing in the problem. The symbols 1 and 2 denote the vectogram tubes of the first and second players (the sets $\mathcal{P}$ and $\mathcal{Q}$, correspondingly)

Example 1. The dynamics is of the following form:

$$
\begin{aligned}
& \ddot{x}+2 \dot{x}=u, \\
& \ddot{y}+0.2 \dot{y}+y=v, \quad x, y \in R^{2}, \quad u \in P, \quad v \in Q .
\end{aligned}
$$

The first player governs an inertial point in the plane. The second object is a twodimensional mathematical pendulum. Both objects have friction proportional to their velocities. The constraints on the players control are taken as the following 


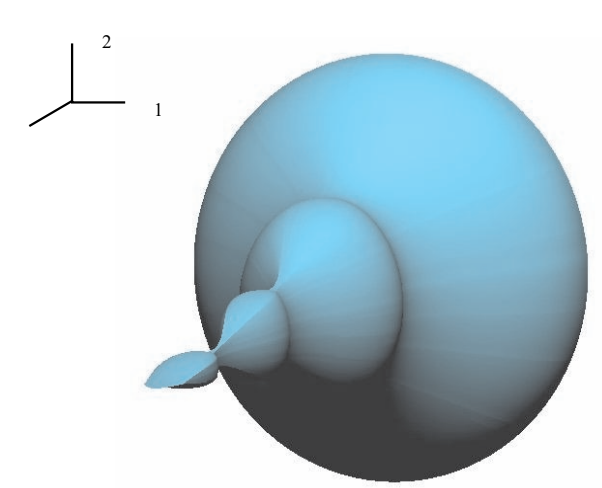

Figure 10: Example 1. A broken level set close to the critical one, $c=2.45098$

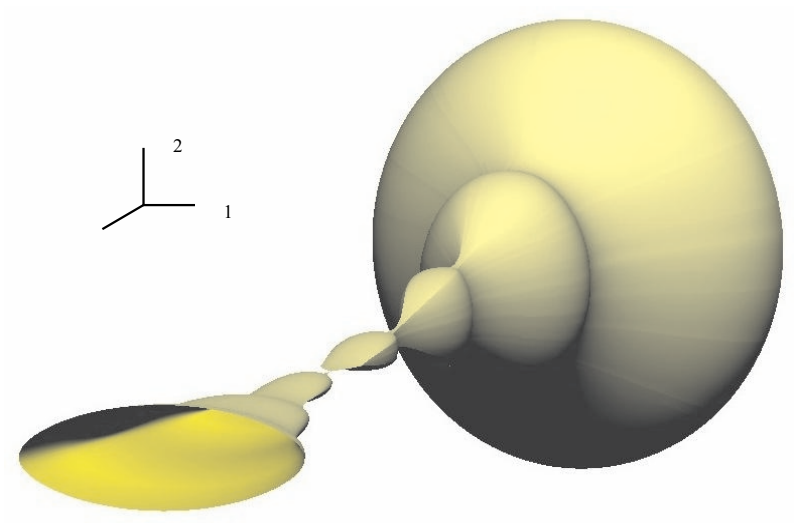

Figure 11: Example 1. General view of the level set with a narrow throat, $c=$ 2.45100

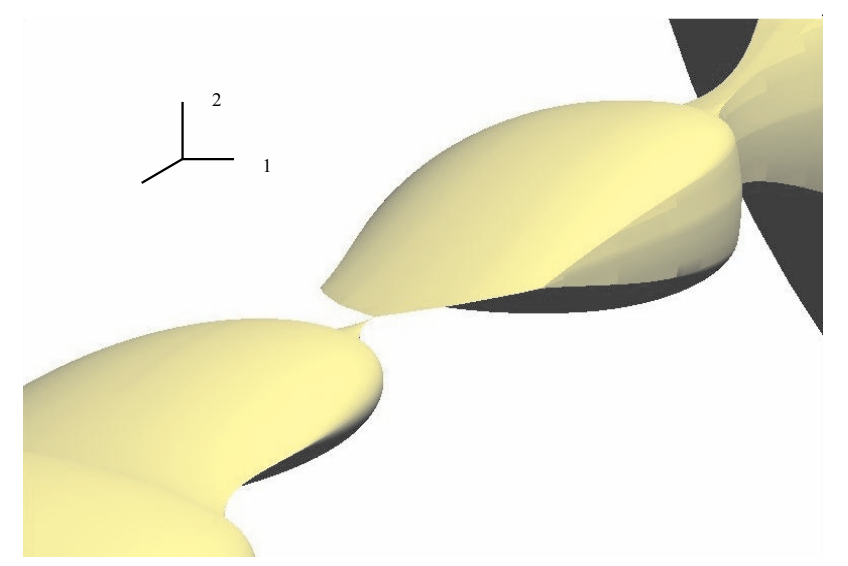

Figure 12: Example 1. Enlarged view of the narrow throat 
ellipses:

$$
P=\left\{u \in R^{2}: \frac{u_{1}^{2}}{0.8^{2}}+\frac{u_{2}^{2}}{0.4^{2}} \leq 1\right\}, \quad Q=\left\{v \in R^{2}: \frac{v_{1}^{2}}{1.5^{2}}+\frac{v_{2}^{2}}{1.05^{2}} \leq 1\right\} .
$$

In Figure 9, the tubes of the players' vectograms $\mathcal{P}(t)$ and $\mathcal{Q}(t)$ are shown. Since the second player dynamics is oscillating, the advantage changes in time. At the beginning of the backward time the second player has complete advantage, but for sufficiently large values of the backward time $\tau$, the advantage shifts to the first player. Enlarged fragment of the vectograms is shown in Figure $9 \mathrm{~b}$.

Figure 10 contains a level set $\boldsymbol{W}_{c}$ (maximal stable bridge) for $c=2.45098$. This bridge breaks (is finite in time). Before the break, the elongation of the subsequent time sections of the tube is changing its direction in reverse time. Namely, before the last throat, the sections are elongated vertically, and after the throat they are elongated horizontally. Changing the orientation happens due to delicate interaction of the players' vectograms $\mathcal{P}(t)$ and $\mathcal{Q}(t)$ similar to the interaction in the problem of pursuit presented in Section 5 .

By slightly increasing the value of $c$ from $c=2.45098$ to $c=2.45100$ the qualitative behavior of the level set suddenly changes. The new level set $(c=$ $2.45100)$ becomes infinite in reverse time and contains also an indifferent zone. This level set is shown in Figure 11. Figure 12 presents an enlarged view of the narrow throat appearing at the instant $\tau=11.95$. This value of the parameter $c$ can be considered as critical one: when $c<2.45100$, the level set breaks, if $c \geq 2.45100$, the break disappears. Adequate reconstruction of the shape of the level set structure needs a very accurate computations for values of $c$ close to the critical one.

This example had been computed for time $\tau \in[0,20]$. Time step $\Delta=0.05$. Circles approximating level sets $\mathcal{M}_{c}$ of the payoff function were changed by regular 100 -gons. The ellipses $P$ and $Q$ representing the constraints of the players' controls were substituted by polygons with 100 vertices.

Example 2. To get an example where a level set has a number of narrow throats, it is necessary to provide multiple changes of advantage between the players while keeping the advantage of each player for a significant period of time. The simplest way to reach this is to provide both players by oscillating dynamics.

Let the dynamics have the following form:

$$
\begin{aligned}
& \ddot{x}-0.025 \dot{x}+1.3 x=u, \\
& \ddot{y}+y=v, \quad x, y \in R^{2}, \quad u \in P, \quad v \in Q .
\end{aligned}
$$

Constraints for the players' controls are taken as identical ellipses

$$
P=Q=\left\{v \in R^{2}: \frac{v_{1}^{2}}{1.5^{2}}+\frac{v_{2}^{2}}{1.05^{2}} \leq 1\right\} .
$$

Because the sets $P$ and $Q$ coincide, then at any instant the ellipses $\mathcal{P}(t)$ and $\mathcal{Q}(t)$ are homothetic: $\mathcal{P}(t)=|\zeta(t)| \cdot P, \mathcal{Q}(t)=|\eta(t)| \cdot P$. If the first player has advantage, that is, if $|\zeta(t)| \geq|\eta(t)|$, then the convexification process is unnecessary: at such instants the function $\gamma(\cdot, t)$ in formula (4.13) of the discrete backward procedure is convex. And vice versa, if the second player has advantage, that is, if $|\zeta(t)|<|\eta(t)|$, 


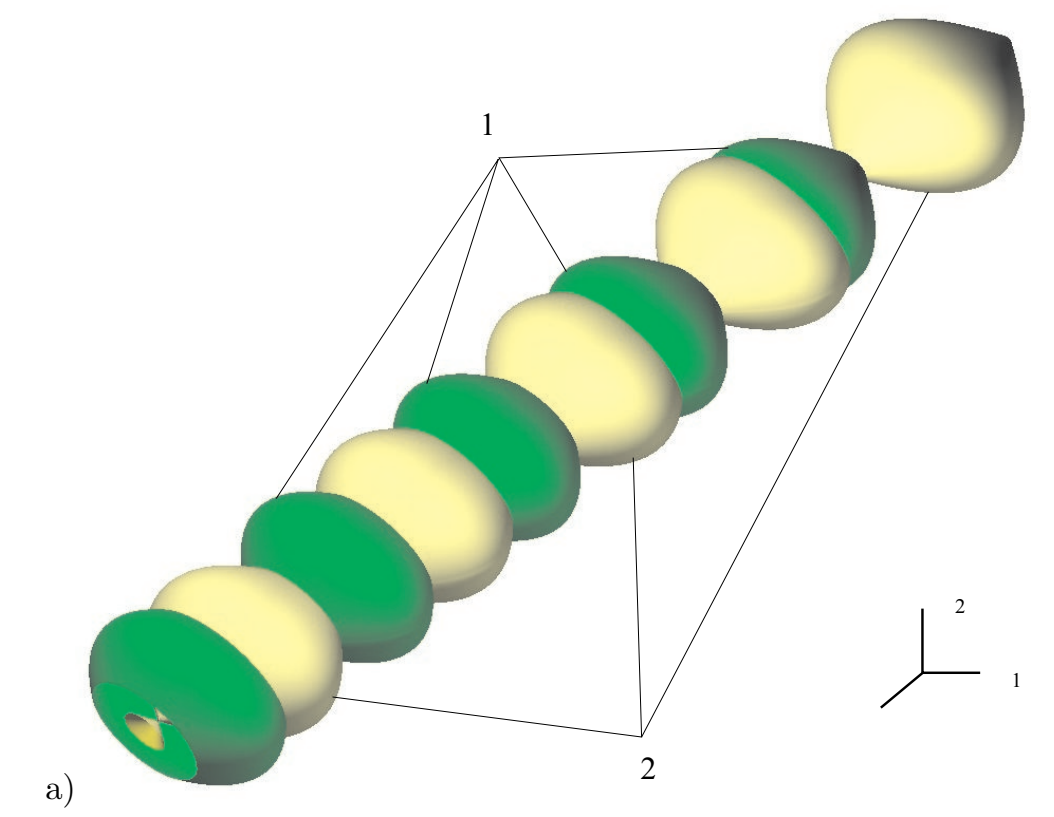

a)

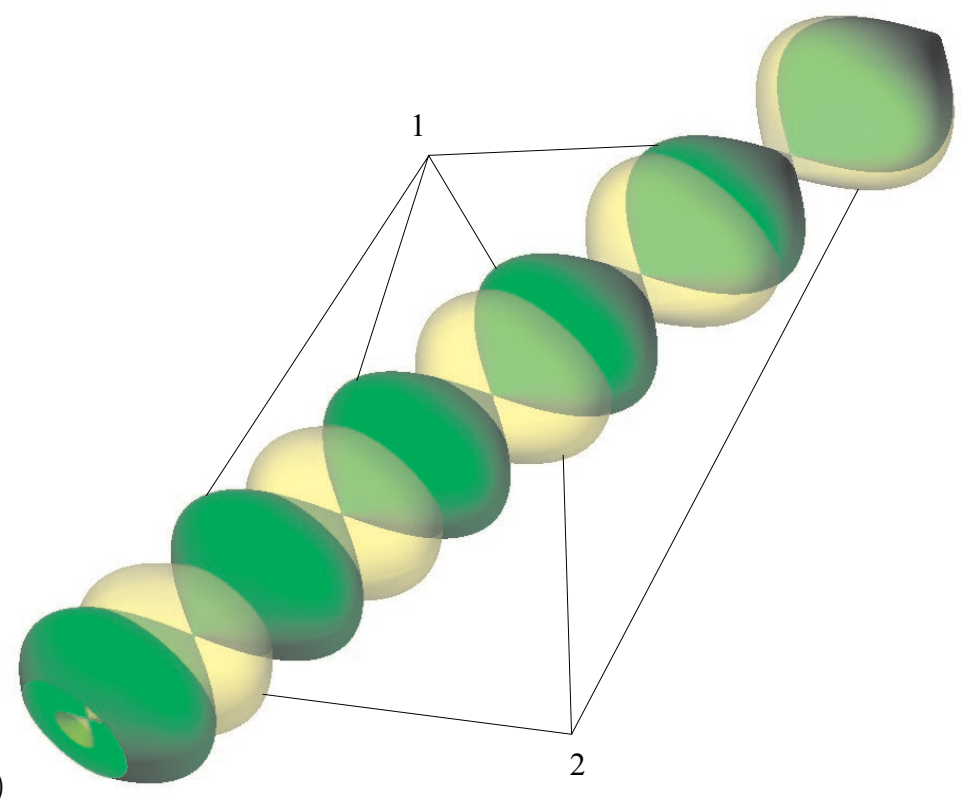

Figure 13: Example 2. General view of the vectogram tubes. The symbols 1 and 2 denote the vectogram tubes of the first and second players (the sets $\mathcal{P}$ and $\mathcal{Q}$, correspondingly). In Figure b), the vectogram tube of the second player is transparent 


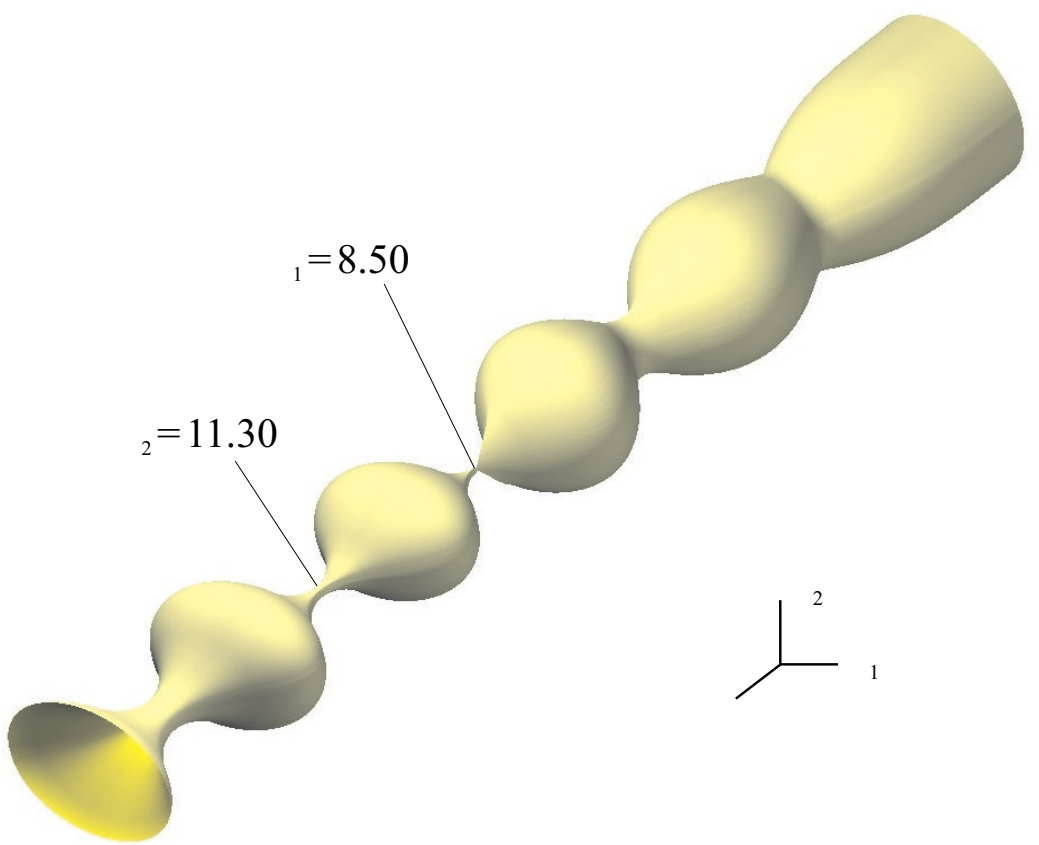

Figure 14: Example 2. General view of the level set with two narrow throats, $c=1.2$. The instants where the throats are the most narrow are shown

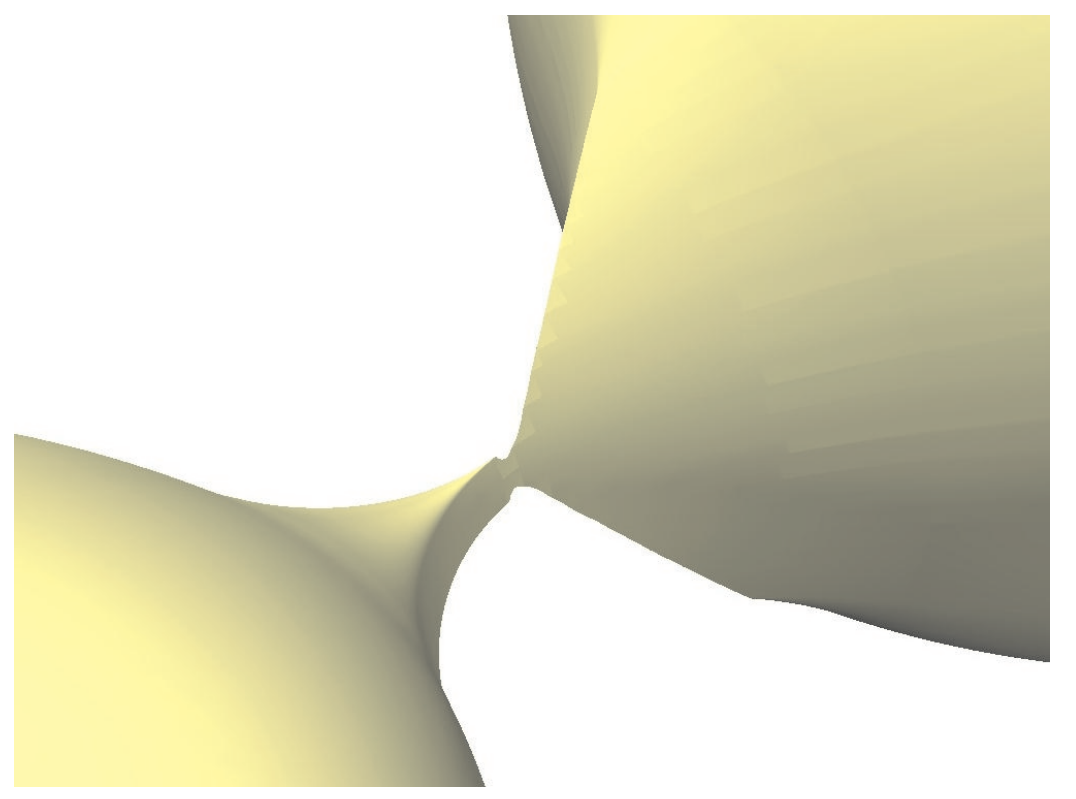

Figure 15: Example 2. Enlerged view of the first narrow throat, $c=1.2$ 
then the function $\gamma(\cdot, t)$ can be non-convex and the convexification process must be applied.

In Figures 13a and 13b, the tubes of players' vectograms are shown. The difference between these figures is that in Figure 13b vectogram tube of the second player is transparent.

Figure 14 shows a general view of the level set $\boldsymbol{W}_{c}$ for $c=1.2$. In Figure 15, an enlarged view of the first narrow throat is presented. The instants, where the throats are most narrow, are $\tau_{1}=8.50$ and $\tau_{2}=11.30$.

This example had been computed for time $\tau \in[0,16]$. Time step $\Delta=0.05$. Near the throats the time step is ten times smaller: $\Delta=0.005$. The number of vertices in the approximating polygons is the same as in the first example.

Note again that despite of the similitude of the players' vectograms (of the sets $\mathcal{P}(t)$ and $\mathcal{Q}(t)$ ), the lack of similitude between the vectograms and sections $\boldsymbol{W}_{c}(t)$ leads to a violation of "monotypicity" of the problem and, consequently, the presence of narrow throats with complicated structure.

Example 3. The dynamics of this example has the form

$$
\begin{aligned}
& \ddot{x}+0.025 \dot{x}+1.2 x=u, \\
& \ddot{y}+0.01 \dot{y}+0.85 y=v, \quad x, y \in R^{2}, \quad u \in P, \quad v \in Q .
\end{aligned}
$$

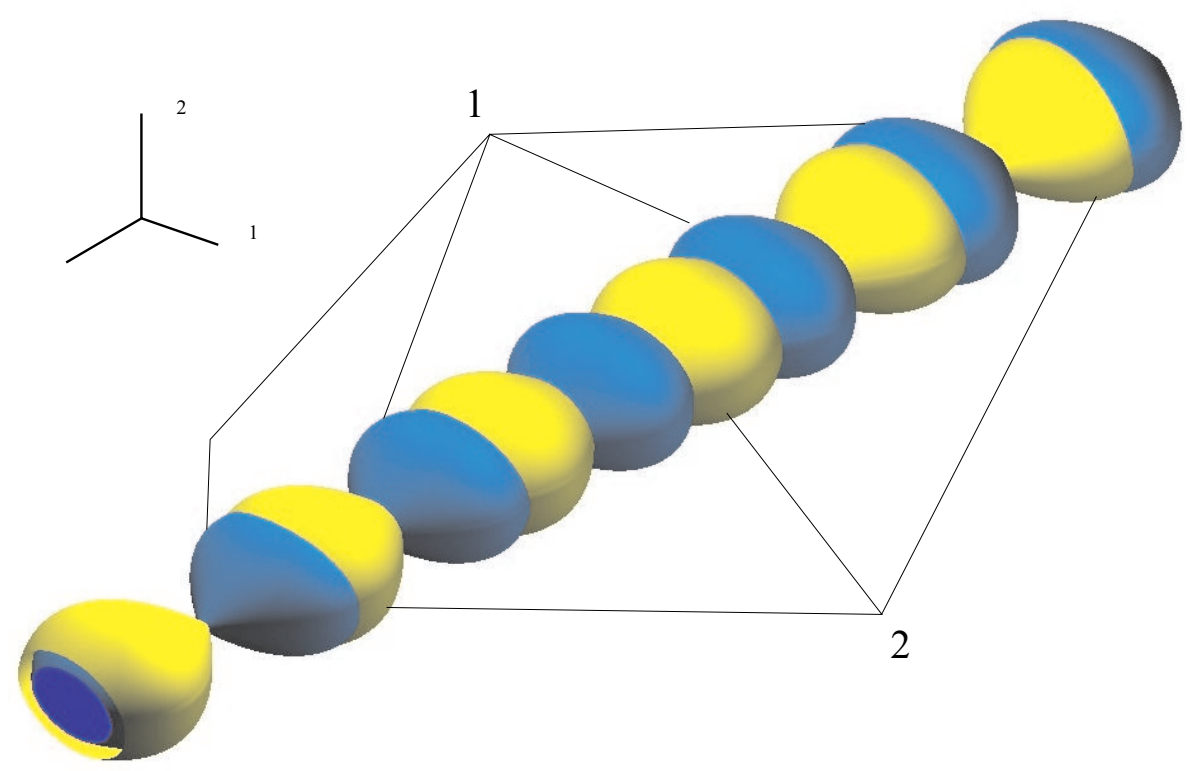

Figure 16: Example 3. General view of the vectogram tubes. The symbols 1 and 2 denote the vectogram tubes of the first and second players (the sets $\mathcal{P}$ and $\mathcal{Q}$, correspondingly) 


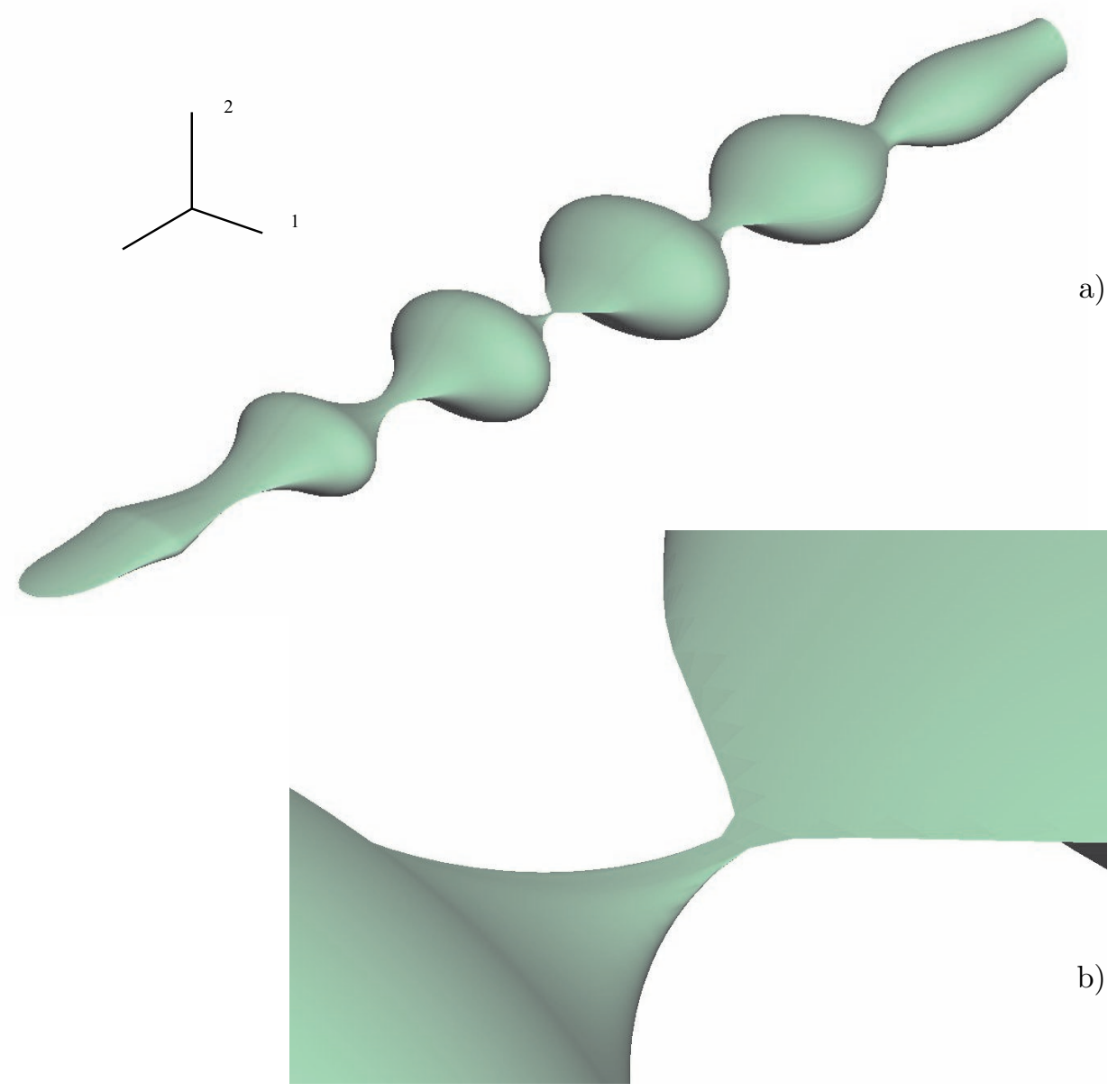

Figure 17: Example 3. a) General view of the level set with three narrow throats, $c=0.397 ; \mathrm{b})$ Enlarged view of the narrowest throat (the middle one)

The constraints are taken as follows:

$$
P=\left\{u \in R^{2}: \frac{u_{1}^{2}}{2.0^{2}}+\frac{u_{2}^{2}}{1.3^{2}} \leq 1\right\}, \quad Q=\left\{v \in R^{2}: \frac{v_{1}^{2}}{1.5^{2}}+\frac{v_{2}^{2}}{1.05^{2}} \leq 1\right\} .
$$

The vectograms of this example are shown in Figure 16. A level set $\boldsymbol{W}_{c}$ of the value function corresponding to $c=0.397$ is drawn in Figure 17a. Three narrow throats are seen. Figure $17 \mathrm{~b}$ presents an enlarged view of the most narrow throat (the middle one).

\section{Summary and Concluding Remarks}

The paper presented four examples of linear zero-sum differential games with bounded controls, fixed terminal time and terminal cost. The cost function in all 
of these examples is a convex function of only two components of the state vector, allowing to transform the original game to a two-dimensional "equivalent" game, which is much more convenient for analysis.

All of the selected examples exhibit the phenomenon of "narrow throats" of the level set of the value function. This phenomenon is closely connected to the existence of "indifferent" zones in the equivalent game space, where the value function is constant and the optimal controls are arbitrary. Such indifferent zones can exist only if relation of players' vectograms in the equivalent game is such that none of the players has a complete advantage during the entire game. If the control constraints (and, consequently, the vectograms) have circular symmetry, the indifferent zone (bounded by the "critical" level set of the value function) starts in reverse time at a single point on the time axis and the neighborhood of the point is called the throat. Depending on the number of advantage changes, there can be more than one throats. Repeated advantage changes can be the result of oscillatory dynamics of one or both players. If the circular symmetry of the control constraints is perturbed, as in the case of elliptical vectograms found in all the selected examples, the time sections of the critical level set near the throat becomes elongated in one direction. Contraction of the time sections of a level set in direct time indicates the advantage of the minimizing player, while expansion indicates the advantage of the maximizer. The change in the orientation of the time sections of the level sets near the narrow throat indicates the shift of advantage in the components of their vectograms. This last phenomenon may have great importance in the development of optimal evasive maneuvers from guided missiles.

The discovery and the analysis of such complex behavior could be achieved only by an intricate and very accurate computational algorithm for construction of the level sets of the value function, developed by the first two authors in a multi-year effort.

\section{Acknowledgment}

This work is supported by Russian Foundation for Basic Research with grants No. 03-01-00415, No. 02-01-96424, and No. 01-07-90210.

\section{References}

Averbukh V.L., S.S. Kumkov, E.A. Shilov, D.A. Yurtaev, and A.I. Zenkov (1999). "Specialized scientific visualization systems for optimal control application". A Proceedings Volume from the IFAC Workshop on Nonsmooth and Discontinuos Problems of Control and Optimization, Chelyabinsk, Russia, 17-20 June 1998, Batukhtin V.D., F.M. Kirillova, and V.I. Ukhobotov (Eds.), Pergamon Press, Great Britain, $143-148$.

Bardi, M. and I.C. Dolcetta (1997). Optimal Control and Viscosity Solutions of HamiltonJacobi-Bellman Equations, Birkhäuser: Boston.

Botkin, N.D. (1982). "Evaluation of numerical construction error in differential game with fixed terminal time". Problems of Control and Information Theory, No.11, $283-295$.

Botkin, N.D. and M.A. Zarkh (1984). "Estimation of error for numerical constructing absorption set in linear differential game". Algorithms and programs for solving linear 
differential games, A.I Subbotin and V.S. Patsko (Eds.), Sverdlovsk: Institute of Mathematics and Mechanics, 39-80. (in Russian).

Cardaliaguet, P., M. Quincampoix, and P. Saint-Pierre (1999). "Set-Valued Numerical Analysis for Optimal Control and Differential Games". Annals of the International Society of Dynamic Games, Vol.4, M. Bardi, T.E. Raghavan, and T. Parthasarathy (Eds.), 177-247.

Grigorenko, N.L., Yu.N. Kiselev, N.V. Lagunova, D.B. Silin, and N.G. Trin'ko (1993). "Methods for solving differential games". Mathematical modelling. Moscow, Moscow State University, 296-316. (in Russian).

Isakova, E.A., G.V. Logunova, and V.S. Patsko (1984). "Computation of stable bridges for linear differential games with fixed time of termination". Algorithms and programs for solving linear differential games, A.I Subbotin and V.S. Patsko (Eds.), Sverdlovsk: Institute of Mathematics and Mechanics, 127-158. (in Russian).

Krasovskii, N.N. and A.I. Subbotin (1988). Game-theoretical control problems. New York: Springer-Verlag.

Kumkov, S.S., V.S. Patsko and J. Shinar (2000). "Level Sets of the Value Function in Linear Differential Games with Elliptical Vectograms". Proceedings of the 11th IFAC Workshop "Control Applications of Optimization" (CAO 2000), July 3-6, 2000, St.Petersburg, Russia, Vol. 2, V.Zakharov, (Ed.), Pergamon Press, Oxford, 579-584.

Kumkov, S.S. and V.S. Patsko (2000). "Maximal stable bridges in the L.S.Pontryagin's example". Vestnik Udmurtskogo Universiteta. Matematika, Mekhanika, No. 1, 92103. (in Russian)

Kurzhanski, A.B. and I. Valyi (1997). Ellipsoidal Calculus for Estimation and Control. Birkhauser, Boston.

Isaacs, R. (1965). Differential games. John Wiley and Sons, New York.

Melikyan, A.A. and J. Shinar (2000). "Identification and construction of singular surface in pursuit-evasion games". Annals of the International Society of Dynamic Games, Vol. 5, J.A. Filar and V. Gaitsgory (Eds.), 151-176.

Mezentsev, A.V. (1972). "A class of differential games". Engrg. Cybernetics, Vol. 9, No. 6, 975-978. Transl. from Izv. Akad. Nauk SSSR. Tekhn. Kibernet., No. 6, 1971, 3-7. (in Russian).

Nikol'skii, M.S. (1984). The first direct method of L.S.Pontryagin in differential games. Moscow: Moscow State Univ. (in Russian).

Patsko, V.S. (1996). "Special Aspects of Convex Hull Constructing in Linear Differential Games of Small Dimension". Control Applications of Optimization 1995. A Postprint Volume from the IFAC Workshop, Haifa, Israel, 19-21 December 1995, Pergamon, 19-24.

Polovinkin, E.S., G.E. Ivanov, M.V. Balashov, R.V. Konstantiov, and A.V. Khorev (2001). "An algorithm for the numerical solution of linear differential games". Matematicheskic sbornik, Vol.192, No.10, 95-122. (in Russian). Transl. as Sbornik: Mathematics, Vol.192, No. 10, 1515-1542.

Ponomarev, A.P. and N.H. Rozov (1978). "Stability and convergence of the Pontryagin's sums". Vestnik Moskov. Univ., Ser. Vyčisl. Mat. Kibernet., No. 1, 82-90. (in Russian).

Pontryagin, L.S. (1972). "Linear differential games". International Congress of Mathematics, Nice, 1970. Reports of Soviet Mathematics. Moscow: Nauka, 248-257. (in Russian).

Pschenichnyi, B.N. and M.I. Sagaidak (1970). "Differential games of prescribed duration". Cybernetics, Vol.6, No. 2, 72-80. (in Russian). 
Shinar, J., M. Medinah and M. Biton (1984). "Singular surfaces in a linear pursuitevasion game with elliptical vectograms". Journal of Optimization Theory and Optimization, Vol. 43, No. 3, 431-458.

Shinar, J. and M. Zarkh (1996). "Pursuit of a faster evader - a linear game with elliptical vectograms". Proceedings of the Seventh International Symposium on Dynamic Games, Yokosuka, Japan, 855-868.

Subbotin, A.I and V.S. Patsko (Eds.) (1984). Algorithms and programs for solving linear differential games. Sverdlovsk: Institute of Mathematics and Mechanics. (in Russian).

Taras'ev, A.M., V.N. Ushakov, and A.P. Khripunov (1988). "On a Computational Algorithm for Solving Game Control Problems". J. Appl. Math. Mech., Vol.51, No. 2, 167-172. Transl. from Prikl. Mat. Mekh., Vol.51, No. 2, 1987, 216-242. (in Russian).

Ushakov, V.N. (1981). "On the problem of constructing stable bridges in a differential pursuit-evasion game". Engrg. Cybernetics, Vol. 18, No.4, 16-23. Transl. from Izv. Akad. Nauk SSSR. Tekhn. Kibernet., No.4, 1980, 29-36. (in Russian). 


\section{Appendix}

The following text and figures had not been included to the original paper, but were demonstrated during the presentation made in Goslar on May, 19, 2003.

Now an another example of the problem discussed in Section 5 will be considered. The main difference between this example and the one presented in Subsection 5.2 is that in this case the velocity of the pursuer is greater than the velocity of the evader. This implies some change in the geometry of the nominal collision as it is shown:

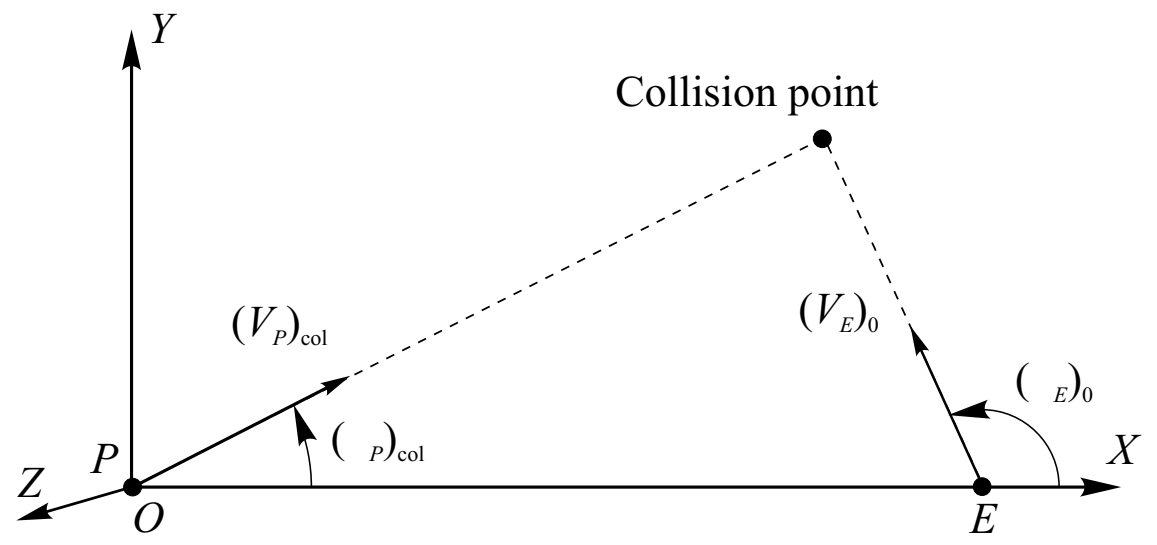

The layout of the ellipses of the control constraints is changed. In Subsection 5.2, the eccentricity of the ellipse $P$ was greater than the eccentricity of $Q$ (figure a), but now the eccentricity of $P$ is less than the eccentricity of $Q$ (figure b):

a)

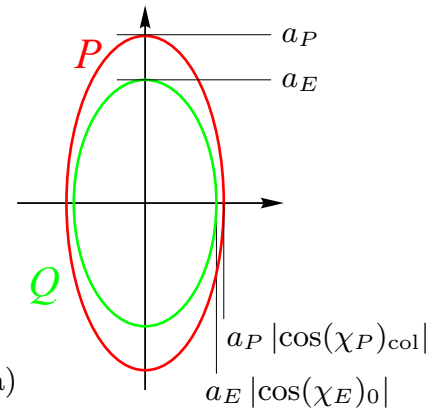

b)

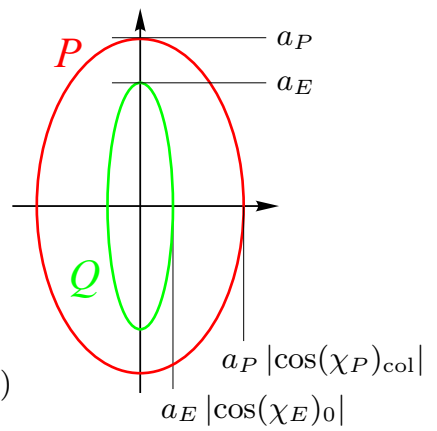

The numerical data of the example are taken as follows:

$$
\frac{\left(V_{E}\right)_{0}}{\left(V_{P}\right)_{\mathrm{col}}}=0.666, \quad \frac{a_{P}}{a_{E}}=5.0, \quad\left|\cos \left(\chi_{P}\right)_{\mathrm{col}}\right|=0.87, \quad\left|\cos \left(\chi_{E}\right)_{0}\right|=0.66, \quad \tau_{P}=1,
$$

that is, the ellipses of the players' controls constraints are

$$
P=\left\{u \in R^{2}: \frac{u_{1}^{2}}{0.87^{2}}+\frac{u_{2}^{2}}{1.00^{2}} \leq 5.0^{2}\right\}, \quad Q=\left\{v \in R^{2}: \frac{v_{1}^{2}}{0.66^{2}}+\frac{v_{2}^{2}}{1.00^{2}} \leq 1\right\} .
$$

The example corresponds to those ones investigated in the papers (Shinar, Medina and Biton, 1984) and (Melikyan and Shinar, 2000). 
Here the vectogram tubes for this example are shown. The first player's tube is cyan, the second one's is magenta:

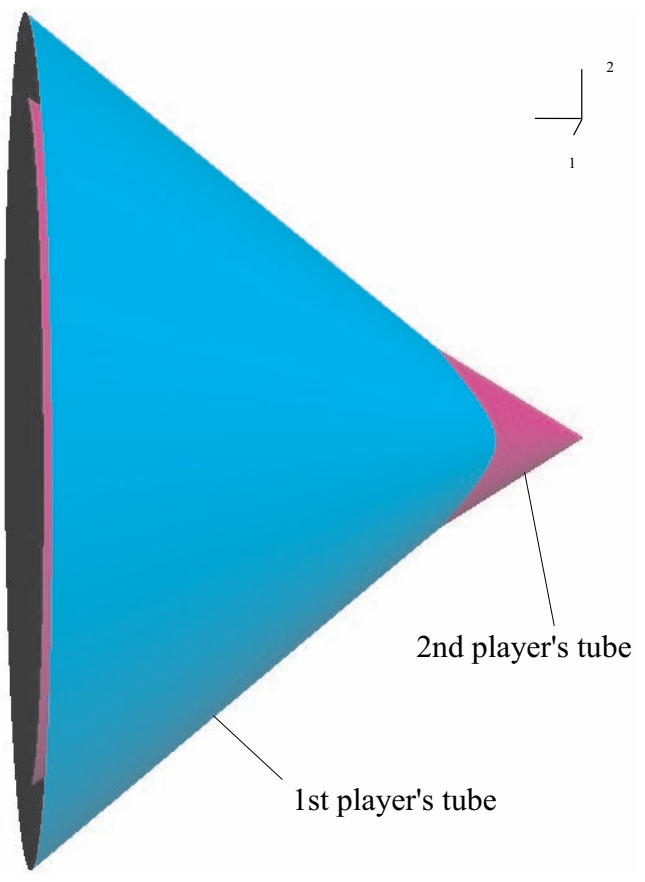

The same tubes are shown from another point of view.

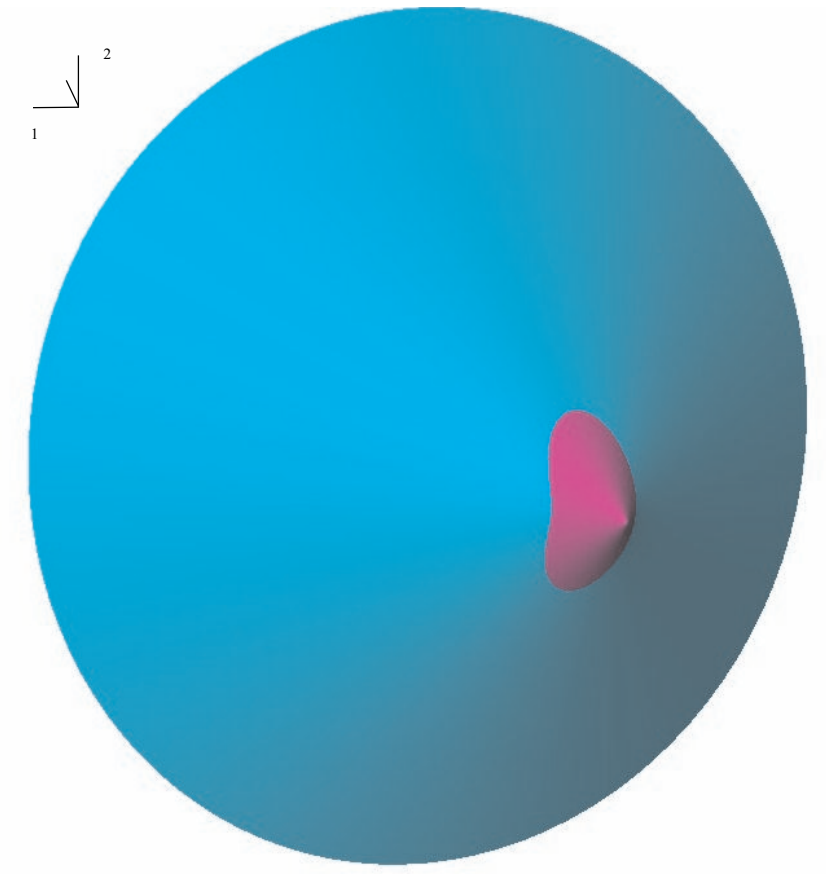


An enlarged part of the previous picture can be seen. On the vectogram tube of the second player the contours of some its time sections are shown:

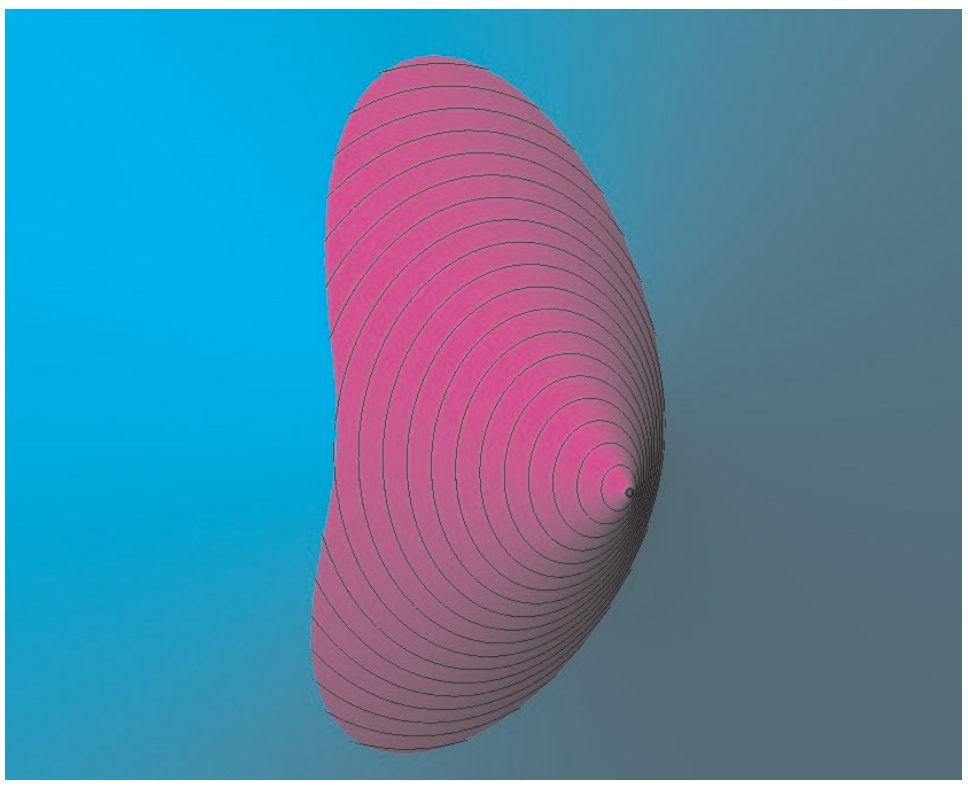

In comparison with the case of the slower pursuer, one can see that as the backwards time $\tau$ grows the first player gets the advantage in the horizontal direction at first, before gaining a complete advantage. This affects the configuration of the time sections of the level set near the narrow throat.

This figure contains a level set close to the critical one:

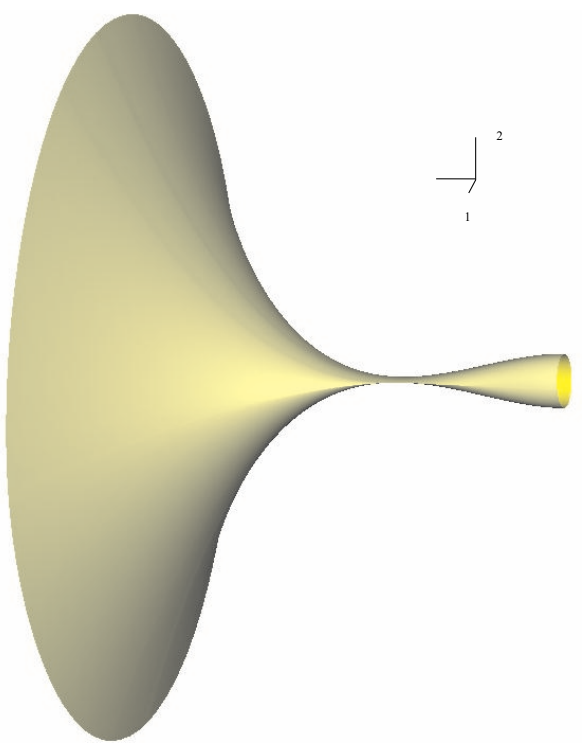

The critical value $\bar{c}=0.141$. Backward instant of the narrow throat $\tau^{*}=0.925$. 
A close view of the narrow throat is shown. One can see that the orientation of the time sections' elongation does not change. Both before the throat, and after it, the sections are elongated horizontally.

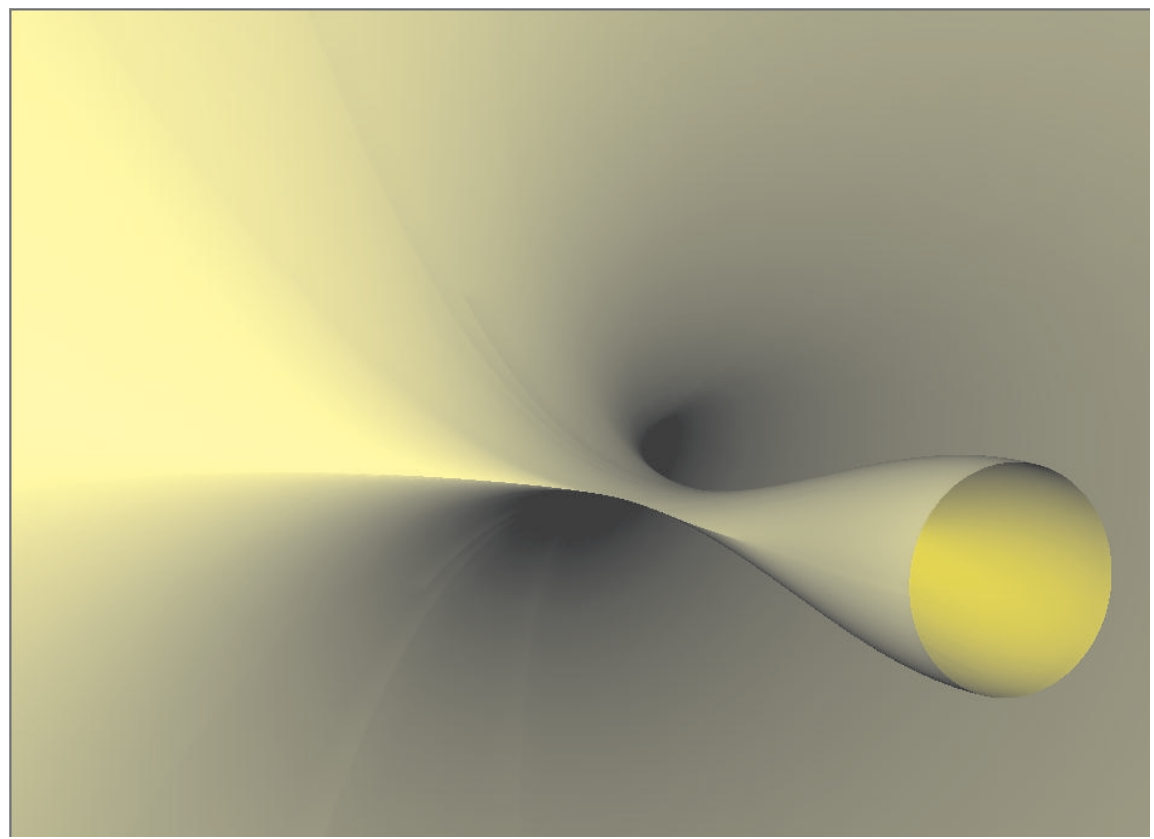

The previous picture is repeated with contours of some time sections of the level set.

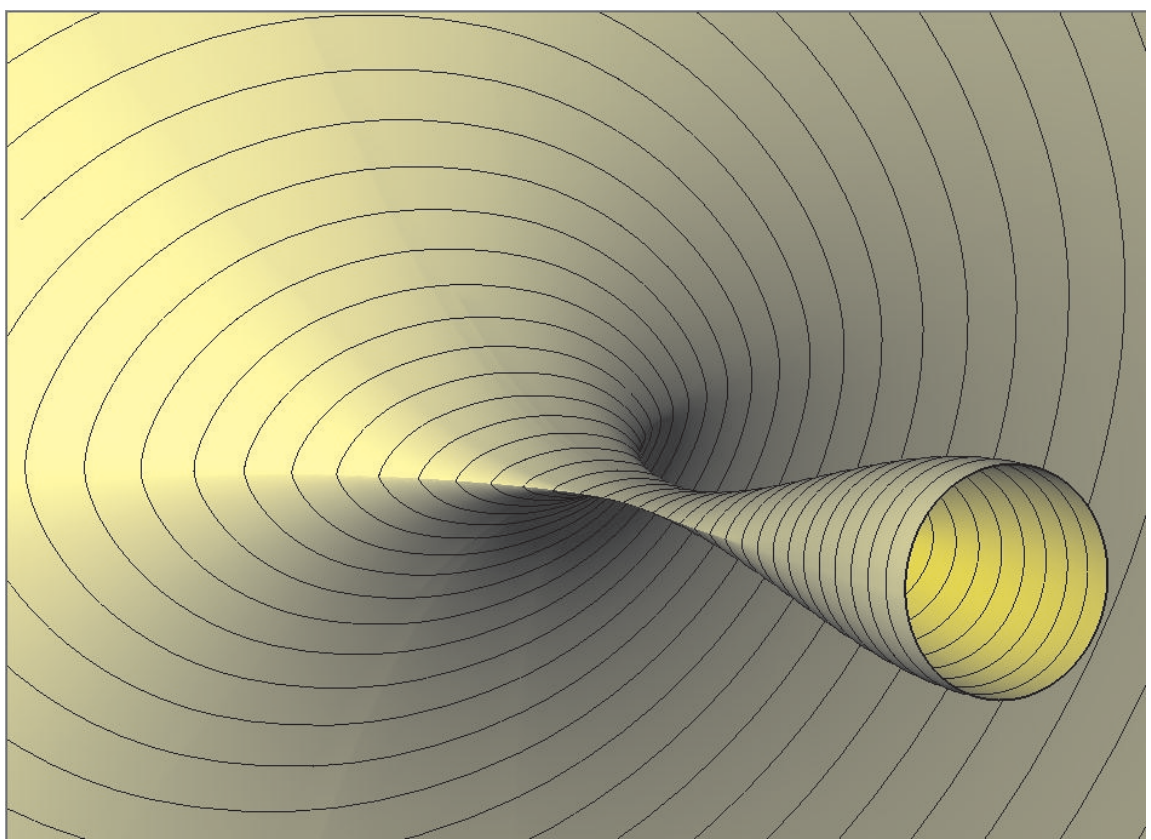


This figure shows again a view of the vectogram tubes. The second player's vectogram is transparent.

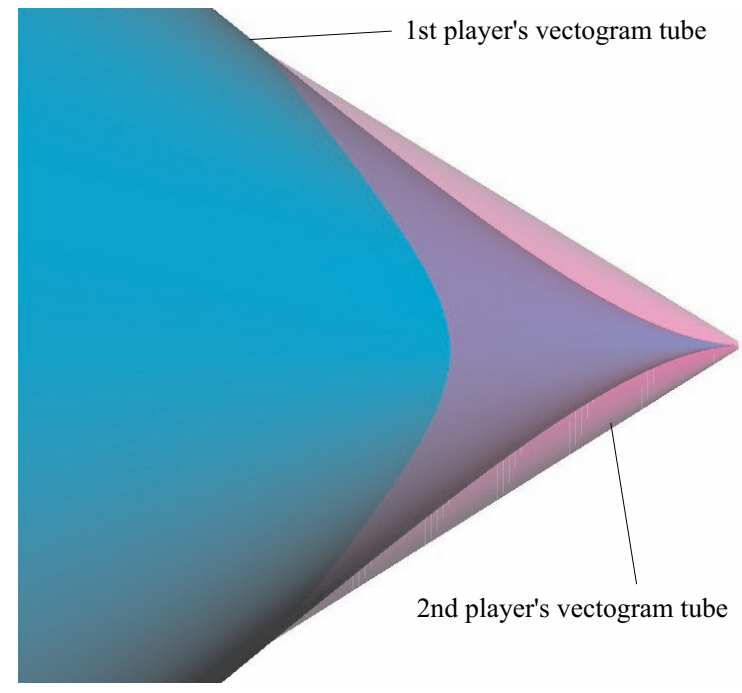

The backward time axis goes from right to left, and thee axis $\xi_{2}$ is directed upwards. The axis $\xi_{1}$ is orthogonal to the sheet.

Using the point of view of the previous figure, a scene is given containing also the level set close to the critical one.

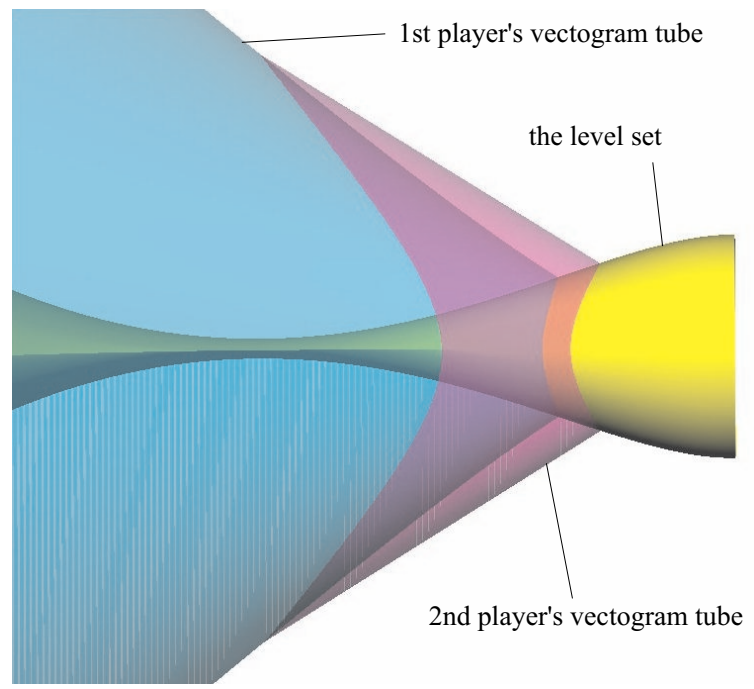

Both vectograms tubes are transparent now. Such an overlap demonstrates clearly the influence of the players' vectograms on the geometry of the level set surface. For example, one can easily see that when the first player gains complete advantage the narrow throat ends (the tube of the level set starts to enlarge). In addition, it is seen that before that instant the tube contracts due to the advantage of the second player. 